\title{
Bauernsohn, Erfinder, Katholik: Jacob Mayer (1813-1875)*
}

\author{
Von Edwin ERnst Weber
}

Neben dem Bildhauer Landolin Ohnmacht ${ }^{1}$ ist der Erfinder und Industriepionier Jacob Mayer zweifellos die über die Grenzen von Ortschaft und Region hinaus bekannteste Persönlichkeit aus Dunningen bei Rottweil. Sowohl in seinem südwestdeutschen Herkunftsraum wie auch in seinem rheinisch-westfälischen Wirkungskreis zählt Jacob Mayer zu den „großen Söhnen“, dem bereits mehrere Kurzbiografien gewidmet wurden und der in der Industriegeschichte des Ruhrgebiets einen prominenten Rang beanspruchen kann ${ }^{2}$. Obwohl Jacob Mayer mithin in zahlreichen Büchern und neuerdings auch im Internet ${ }^{3}$ präsent ist, wissen wir zwar viel über die industriegeschichtliche Bedeutung seiner Erfindungen sowie die Entwicklung des von ihm mitbegründeten Betriebs in Bochum, letztlich aber wenig über seine Persönlichkeit und sein Denken.

"Geringfügig überarbeitete und mit Anmerkungen versehene Fassung eines Festvortrags vom 10. Mai 2013 in Dunningen zum 200. Geburtstag von Jacob Mayer.

${ }^{1}$ Julius Wilbs, Landolin Oh(n)macht. Bildhauer. 1760-1834, in: Gerhard TAdDEy/Joachim Fischer (Hg.), Lebensbilder aus Baden-Württemberg, Bd. 20, Stuttgart 2001, S. $146-$ 165; Mechtild Oнммаснт, Landelin (!) Ohnmacht, Lindenberg im Allgäu 2013.

2 Die wichtigsten biografischen Annäherungen: W(alter) Bertram, Jacob Mayer. Der Erfinder des Stahlformgusses. Zur 125. Wiederkehr seines Geburtstages am 1. Mai 1938, Berlin 1938; Eugen ReinerT, Jakob (!) Mayer. Erfinder des Stahlformgusses und Begründer des Bochumer Vereins. 1813-1875, in: Schwäbische Lebensbilder, Bd.2, hg. von Hermann Haering, Stuttgart 1941, S.340-350; Theodor Heuss, Jacob Mayer, in: Ders., Deutsche Gestalten. Studien zum 19. Jahrhundert, Stuttgart/Tübingen 1947, S.154-161; Adalbert Frensdorff, Jacob Mayer, in: NDB, Bd.16, Berlin 1990, S. 543 f. Stellvertretend für die wirtschaftsgeschichtlichen Darstellungen seien genannt: Walter DÄBRITZ, Bochumer Verein für Bergbau und Gußstahlfabrikation in Bochum. Neun Jahrzehnte seiner Geschichte im Rahmen der Wirtschaft des Ruhrbezirks, Düsseldorf 1934; Günter OGger, Die Gründerjahre. Als der Kapitalismus jung und verwegen war, München 1982; Marco Rudzinski, Ein Unternehmen und „seine“ Stadt. Der Bochumer Verein und Bochum vor dem Ersten Weltkrieg (Veröffentlichungen des Instituts für soziale Bewegungen, Schriftenreihe A: Darstellungen, Bd. 51), Essen 2012.

${ }^{3}$ Als Beispiele: Wikipedia (Jacob Mayer(Fabrikant)); Internet-Auftritt der Stadt Bochum (www.bochum.de - Historische Bochumer Persönlichkeiten); Internet-Auftritt der JacobMayer-Schule Dunningen (www.jacob-mayer-schule.de - Geschichte/Namensgeber).

Zeitschrift für Württembergische Landesgeschichte 74 (2015), S. 173-200.

(C) Kommission für geschichtliche Landeskunde in Baden-Württemberg und Württembergischer

Geschichts- und Altertumsverein e.V.

ISSN 0044-3786 
Dies liegt zum einen am vorrangig technikgeschichtlichen Blickwinkel der bisherigen Forschung zu Leben und Werk Mayers, zum anderen aber auch am weitgehenden Fehlen von Selbstzeugnissen. Wie viele Persönlichkeiten aus der Technik hat auch Jacob Mayer keine Aufzeichnungen über sein Leben ${ }^{4}$ und eine lediglich schmale Korrespondenz - namentlich mit seinem in Dunningen verbliebenen ältesten Bruder Josef Mayer - hinterlassen ${ }^{5}$ und stützt sich unser Wissen zu Person und Werdegang jenseits seiner erfinderischen und unternehmerischen Aktivitäten vorrangig auf Schilderungen und Deutungen Dritter und hier insbesondere auf die seines Neffen Peter Josef Sieber aus Trier ${ }^{6}$. Auch kursieren verschiedene Legenden zumal aus den Lehr- und Wanderjahren Mayers - etwa von der heimlichen Beobachtung seines Kölner Lehrmeisters bei dessen vergeblichen Versuchen zur Gussstahlherstellung durch ein in den Kammerboden gebohrtes Guckloch oder von der angeblichen Flucht unter Lebensgefahr aus England vor den ihm nachstellenden Arbeitskollegen -, deren Wahrheitsgehalt sich nur schwerlich nachweisen lässt ${ }^{7}$.

Diese Studie unternimmt den Versuch, den Blick auf Jacob Mayer auf drei Feldern zu erweitern: Zum einen gilt das sozialgeschichtliche Interesse seinem dörflichen und familiären Hintergrund mit den bis in die Bochumer Zeit wirksamen Dunninger Verwandtschaftsbeziehungen; zum anderen gilt das Interesse Mayers Erfolg als Erfinder und seinem gleichzeitigen Scheitern als Unternehmer; und zum dritten schließlich erscheint die katholische Prägung und Verankerung Jacob Mayers als aufschlussreich für das Verständnis seiner Persönlichkeit. Neben neuerer Literatur zur Stadt- und Industriegeschichte Bochums ${ }^{8}$ stützt sich die Untersuchung vor allem auf Quellen aus dem Gemeinde- sowie dem Pfarrarchiv von Dunningen zu den Verwandtschafts- und Besitzverhältnissen von Jacob Mayers Vorfahren und Geschwistern, weiter auf die erwähnte Korrespondenz des Erfinders mit seinem Bruder Josef in Dunningen aus dem Historischen Archiv Krupp und schließlich auf die Schilderungen und Erinnerungen seines Neffen Peter Josef Sieber.

${ }^{4}$ Reinert (wie Anm. 2) S.349; auch Rudzinsky (wie Anm. 2) S. 86 schreibt von einem „Mangel an originären Quellen“ zu Jacob Mayer.

5 Acht Schreiben von Jacob Mayer aus Köln bzw. Bochum an seinen Bruder Josef Mayer in Dunningen zwischen 1837 und 1844 (Historisches Archiv Krupp Essen WA 80/1423; Kopien Heimatmuseum Dunningen).

${ }^{6}$ Schreiben von P(eter Jos.) Sieber zu Trier an Josef Mayer junior in Dunningen vom 28. Oktober 1904 (Heimatmuseum Dunningen); Schreiben von P(eter Jos.) Sieber zu Trier an Lehrer Karl Schneider in Dunningen vom 20. Mai 1907 betreffend Jacob Mayer (Historisches Archiv Krupp Essen WA 80/1420).

7 Zumindest die Kölner Gucklochgeschichte scheint auf die Schilderung von Peter Josef Sieber zurückzugehen (vgl. Schreiben Sieber von 1904, wie Anm.6). Von Bertram (wie Anm. 2) S. 4 werden beide Geschichten erstmals publiziert.

8 Rudzinsky (wie Anm.2); Enno Neumann, Ein Grabdenkmal erzählt Bochumer Geschichte. Jacob Mayer und der Bildhauer Wilhelm Gardy, in: Johannes Volker Wagner (Hg.), Das Stadtarchiv. Schatzkammer - Forschungsstätte - Erlebnisort, Essen 2004, S. 145156.

Zeitschrift für Württembergische Landesgeschichte 74 (2015), S. 173-200.

(C) Kommission für geschichtliche Landeskunde in Baden-Württemberg und Württembergischer Geschichts- und Altertumsverein e.V.

ISSN 0044-3786 


\section{Der dörfliche und familiäre Hintergrund}

Jacob Mayer wird am 1. Mai 1813 als zweitältestes von fünf Kindern des Kleinbauern Adam Mayer (1772-1833) und seiner ersten Frau Luggard geb. Mauch (1785-1821) in Dunningen geboren ${ }^{9}$. Das einstige Untertanendorf der Reichsstadt Rottweil gehört 1813 seit mittlerweile zehn Jahren dem 1806 zum Königreich aufgestiegenen Württemberg an und ist in dieser Zeit von den Erschütterungen der endlosen Kriege zunächst mit und seit 1813 gegen Napoleon betroffen ${ }^{10}$. Eine württembergische Bestandsaufnahme ermittelt für das Dorf 1802143 Familien und 975 Seelen, 52 Geburten und 30 Todesfälle, 176 Pferde und 632 Stück Hornvieh sowie den Acker- und Wiesenbau neben der Viehzucht als Haupterwerbsquellen ${ }^{11}$. 130 Schulkinder werden von Schulmeister Aloysi Thaler im Gemeindshaus unterrichtet. Bei der Kirchenvisitation 1808 werden bereits 1.044 Seelen gezählt, als Pfarrer wird Augustin Fidel Georg Braunmühl, ein ehemaliger Benediktiner aus dem Kloster Wiblingen bei Ulm, genannt ${ }^{12}$. Pfarrer Braunmühl dürfte Jacob Mayer getauft haben, und bei Lehrer Thaler könnte er seine erste schulische Ausbildung erhalten haben.

Wenn man dem Visitationsbericht von Pfarrer Strobel aus Rottweil von 1818 Glauben schenkt, sind die sittlichen Verhältnisse in Dunningen zur Jugendzeit Jacob Mayers nur wenig ersprießlich ${ }^{13}$ : In der Gemeinde sieht er eine große Verdorbenheit [...] herrschend mit Nachtschwärmerei, unzüchtige $(m)$ Wirthshausbesuch, Trunkenbeit und damit verbundene (n) Schlägerë̈en, verabredete (n) nächtliche (n) Zusammenkünfte (n) der ledigen Pursche (n) in den Kammern der Weibspersonen, sodann nicht seltenen Uneinigkeiten in den Ehen, kleineren Diebereien und gewaltsamen Einbrüchen, einer jüngst aufgeflogenen Gesellschaft von Falschmünzern und schließlich sogar drei Totschlägen innerhalb weniger Jahre. Vor zwei Jahren sei in Dunningen ein eigentlicher Aufstand gegen den Schultheiß erfolgt, wobe y die grösten Unordnungen vorfielen. Den 71 Jahre alten und kränkelnden Pfarrer erachtet der Visitator angesichts der Roheit und der vielen Gebrechen in der Ge-

${ }^{9}$ Familien-Register I zum Kirchenbuch der Pfarrei Dunningen 1808-1909, S. 265 (Diözesanarchiv Rottenburg (künftig: DAR) M 173 B 18).

10 Zum Übergang Dunningens an Württemberg und den innerdörflichen Verhältnissen vgl. Edwin Ernst Weber, Von der Knechtschaft in die Freiheit? Dunningen und Seedorf beim Übergang von der Reichsstadt Rottweil an Württemberg 1802/03, in: Die Brücke. Dunninger Jahrbuch 17 (2002) S. 23-34.

11 „Beantwortung der 83 Fragen quoad Statum Ecclesiasticum, Politicum et oeconomicum in ehem. Obervogteiflecken Dunningen, Seedorf, Herrenzimmern etc." - November 1802 (StAL D 23 Nr. 29).

12 „Tabellarischer Ausweis ueber die Dekanatsvisitation vom 22. August bis 14. Oktober 1808 in verschiedenen Wirtembergische(n) Gemeinden des Landkapitels Rotweil“von Dekan Dr. Ludwig Anton Haßler - Dunningen (DAR A I 2 b Nr. 138).

13 Spezial-Bericht vom 29. Juli 1818 über die in Dunningen am 19. Mai 1818 abgehaltene Kirchenvisitation durch Visitator Strobel, Rottweil (DAR G 1.8 Nr. 494). 
meinde als ebenso überfordert und ablösungsbedürftig wie den Schultheißen, der nicht nur untätig sei, sondern auch nicht das Vertrauen seiner Mitbürger habe.

Jacob Mayer ist der Spross einer alteingesessenen Bauernfamilie, deren Vorfahren sich in Dunningen bis in das 17. Jahrhundert zurückverfolgen und - wie etwa die als Schultheiß bzw. Dorfvogt genannten Urgroßväter Christian Mayer (17071743) und Matthäus Graf (1706-1763) ${ }^{14}$ - sich vielfach in gemeindlichen Führungsämtern nachweisen lassen. Sowohl der Großvater Matthäus Mayer (1735-1811) wie auch der Vater Adam Mayer tauchen in den Dunninger Kaufbüchern mit mittelbzw. kleinbäuerlichem Grund- und Viehbesitz und dem Anwesen Nr.136 mit Haus und Scheuer an der Abzweigung der Bitzestraße von der Locherhofer Straße auf $^{15}$.

Weist Dunningen im 18. Jahrhundert noch eine ganze Reihe großbäuerlicher Lehenshöfe und eine polare Sozialstruktur mit rund einem Drittel wohlhabender Vollbauern und zwei Drittel landarmer Taglöhner auf ${ }^{16}$, so entwickelt sich in Dunningen im 19. Jahrhundert eine kleinbäuerliche Dorfgesellschaft. Hintergründe für diese tendenzielle soziale Nivellierung sind die sich durchsetzende Realteilung, das sich lockernde Teilungsverbot für den grundherrschaftlich gebundenen Besitz sowie ein nicht zuletzt durch den beträchtlichen Anteil des bäuerlichen Eigengutes ausgesprochen mobiler Grundstücksmarkt.

Wie sich gerade auch am Beispiel der Familie Mayer nachweisen lässt, werden durch die Güterteilung unter den zumeist zahlreichen Kindern die Anwesen stetig kleiner und zur Auszahlung von Erben mitunter durch parzellenweise Verkäufe sogar regelrecht atomisiert. Neben Matthäus Mayer, dem Großvater Jacob Mayers, mit einem mittelbäuerlichen Besitz lässt sich 1772/1773 noch dessen jüngster Bruder Isidor Mayer als gutsituierter Lehensbauer mit einem Hof von 78 Jauchert nachweisen ${ }^{17}$. In der nächsten Generation begegnen neben Jacob Mayers Vater Adam Mayer noch dessen Brüder Thomas Mayer (1764-1839) und Valentin Mayer

\footnotetext{
14 Nachlass Alfons Haigis (1908-1994), Familienregister Dunningen, Bd.6 (StadtA Schramberg) - Auswertung zu Christian Mayer und Matthäus Graf.

${ }_{15}$ Matthäus Mayer bzw. dessen Enkel Simon Mayer und Sohn Adam Mayer werden 1820 mit einem Grundbesitz von 52 Jauchert 11/2 Vierndel 122 Ruthen und einem - bereits 1804 ermittelten - Viehbestand von 2 Pferden, 5 Stieren, 3 Kühen und 1 Kälble aufgeführt (Güterbuch Dunningen, Oberamt Rottweil, Bd.I, fol.585ff., GemeindeA Dunningen Nr.613); zum Grundbesitz von Adam Mayer s. unten.

${ }^{16}$ Edwin Ernst Weber, Städtische Herrschaft und bäuerliche Untertanen in Alltag und Konflikt. Die Reichsstadt Rottweil und ihre Landschaft vom 30jährigen Krieg bis zur Mediatisierung, Rottweil 1992, S. 239, 242, 244.

17 Im Dunninger Urbar von 1772/1773 wird Matthäus Mayer als Bauer mit Eigengut von 19,3 Jauchert, aber ohne Lehensbesitz, sein Bruder Isidor Mayer dagegen mit Lehensgütern von 70,5 Jauchert und Eigenbesitz von 8,25 Jauchert aufgeführt (vgl. Karl SCHNEIDER, Dunningen im Oberamt Rottweil. Beschreibung und Geschichte, Dunningen 1927, masch., S. 1013, 1017). Isidor Mayer hat dabei auch das Bauernlehen der Rottweiler Bruderschaft inne, das im Dunninger Urbar von 1739 noch seinem Vater Christian Mayer zugeschrieben worden war (vgl. ebd., S. 959, 943).
} 


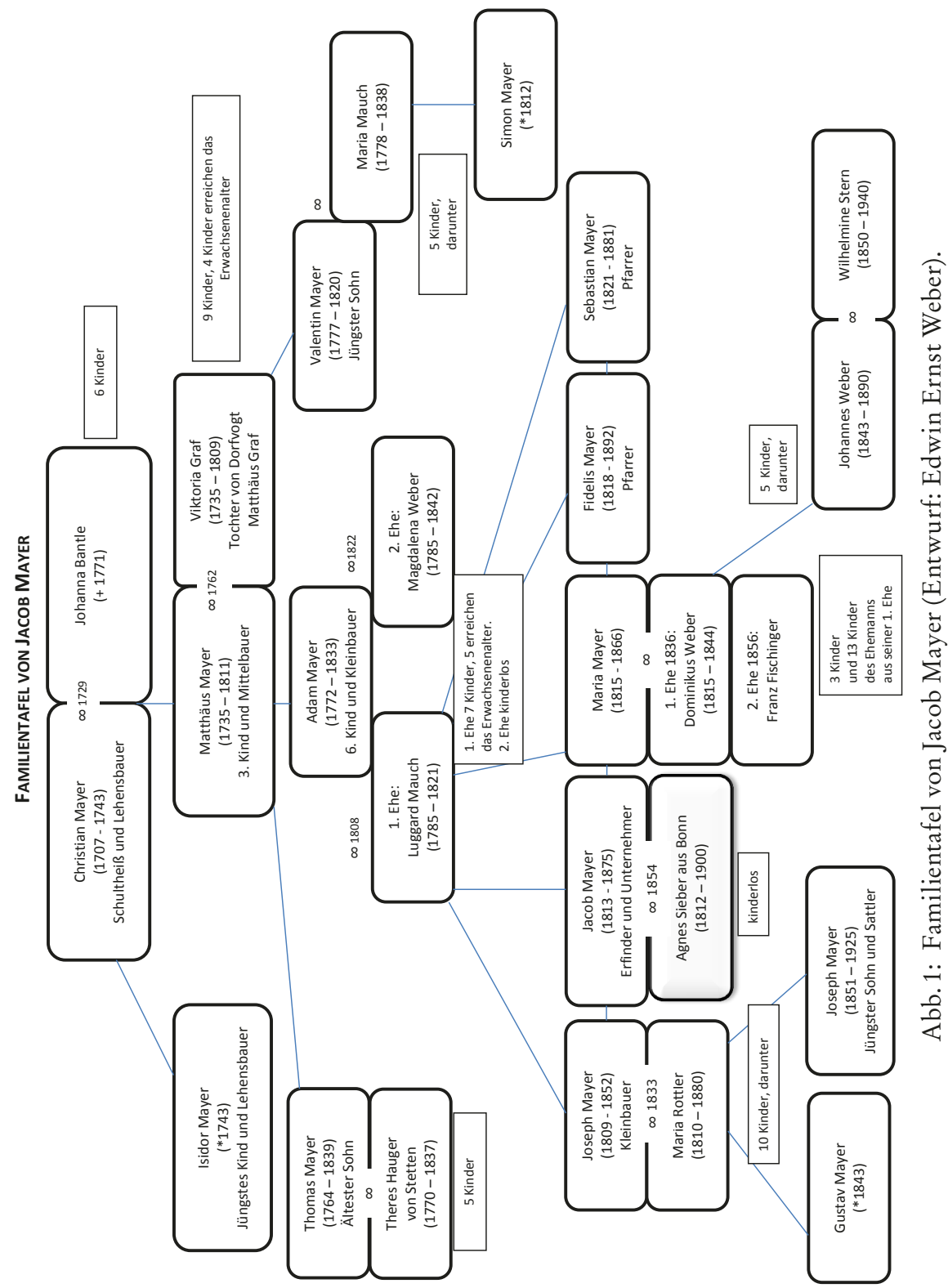

Zeitschrift für Württembergische Landesgeschichte 74 (2015), S. 173-200.

(C) Kommission für geschichtliche Landeskunde in Baden-Württemberg und Württembergischer Geschichts- und Altertumsverein e.V. ISSN 0044-3786 
(1777-1820) mit gleichfalls kleinbäuerlichen Anwesen. Der Hof des verstorbenen Matthäus Mayer mit nunmehr 52,5 Jauchert wird 1820 hälftig zwischen seinem Sohn Adam Mayer und seinem Enkel Simon Mayer, dem Sohn des verstorbenen Valentin Mayer, aufgeteilt ${ }^{18}$.

Im Dunninger Güterbuch taucht Adam Mayer um 1830 mit einem durch parzellenweise Zukäufe erweiterten kleinbäuerlichen Anwesen von immerhin 36,5 Jauchert auf ${ }^{19}$. Seinen an der Abzweigung der Bitzestraße von der Locherhofer Straße gelegenen Hof (Nr.136) gibt er 1820 in einem Tauschgeschäft an seinen jüngeren Bruder Valentin Mayer ab, von dem er wiederum dessen im Graben gelegenes Bauernhaus mit der Gebäudenummer 54 übernimmt ${ }^{20}$. Nur drei Jahre später, am 20. Januar 1823, verkauft Adam Mayer dieses Haus für 1.248 Gulden an den Küfer Leopold Rottler und erwirbt statt dessen vom Wagner Mathias Mauch für 1.435 Gulden das Anwesen Nr. 78 mit Haus, Scheuer, Backhaus und Garten an der Einmündung der Schnurrenstraße in die Dorfbachstraße ${ }^{21}$. Jacob Mayer hat mithin sein vermeintliches Geburtshaus an der Schnurrenstraße erst im Alter von zehn Jahren bezogen, tatsächlich zur Welt gekommen ist er auf der Bitze.

Nach dem Tod des Vaters wird beim Übergang des Anwesens für 2.000 Gulden an den ältesten Sohn Josef Mayer 1833 eine Beschreibung des Bauernhauses erstellt: Das zweistöckige, mit einem Schindeldach versehene Wohn- und Ökonomiegebäude weist zwei Stuben, eine Scheuer, zwei Ställe und einen Schopf auf, unter dem Inventar wird neben zahlreichen landwirtschaftlichen Gerätschaften als einziger Gegenstand der Wohnkultur eine Wanduhr samt Gewicht aufgeführt' ${ }^{22}$.

Aus der am 1. Februar 1808 geschlossenen Eheverbindung von Adam Mayer und der 13 Jahre jüngeren Luggard Mauch gehen insgesamt sieben Kinder hervor: Die Söhne Josef (geb. 28.2.1809), Jacob (geb. 1.5.1813), Fidelis (geb. 12.4.1818) und Sebastian (geb. 20.1.1821) sowie die Tochter Maria (geb. 1.8.1815). Hinzu kommen noch die Zwillinge Maria und Lorenz, die am Tag ihrer Geburt am 3. August 1811 versterben. Acht Monate nach der Geburt ihres jüngsten Sohnes Sebastian stirbt Luggard Mauch im Alter von knapp 36 Jahren. Neun Monate darauf, am 27. Juni 1822, verheiratet sich der fast 50-jährige Adam Mayer wieder mit der gleichfalls 13 Jahre jüngeren Magdalena Weber ${ }^{23}$. Eine relativ rasche Wiederverheiratung verwitweter Männer mit kleinen Kindern ist in vormoderner Zeit weit verbreitet, um die

18 Güterbuch Dunningen, Bd. I (wie Anm. 15) fol. 151v-154r.

19 Güterbuch Dunningen, Oberamt Rottweil, Bd. III, fol.585v-599r (GemeindeA Dunningen Nr.615). Dabei handelt es sich um 8 J 3 V 281 R Öhmdwiesen, verteilt auf 18 Parzellen, und 26 J 1 V 223 R Ackerfeld. In einem Nachtrag werden noch Grundstückserwerbungen durch Adam Mayer von 18 J 21/2 V 258 R aufgeführt.

${ }^{20}$ Ebd.

${ }^{21}$ Ebd.; außerdem Kaufbuch für die Ehrsame Gemeinde Dunningen von Februar 1810März 1824, fol. 213v, 214r (GemeindeA Dunningen Nr. 758).

22 Kaufbuch Dunningen, Oberamt Rottweil, Bd. VI, vom 16. März 1833 bis 2. September 1835 (GemeindeA Dunningen Nr. 763), fol.76r-77v - Kaufvertrag vom 10.7.1833.

${ }^{23}$ Familien-Register I zum Kirchenbuch Dunningen 1808-1909 (wie Anm. 9) S. 265.

Zeitschrift für Württembergische Landesgeschichte 74 (2015), S. 173-200.

(C) Kommission für geschichtliche Landeskunde in Baden-Württemberg und Württembergischer Geschichts- und Altertumsverein e.V.

ISSN 0044-3786 


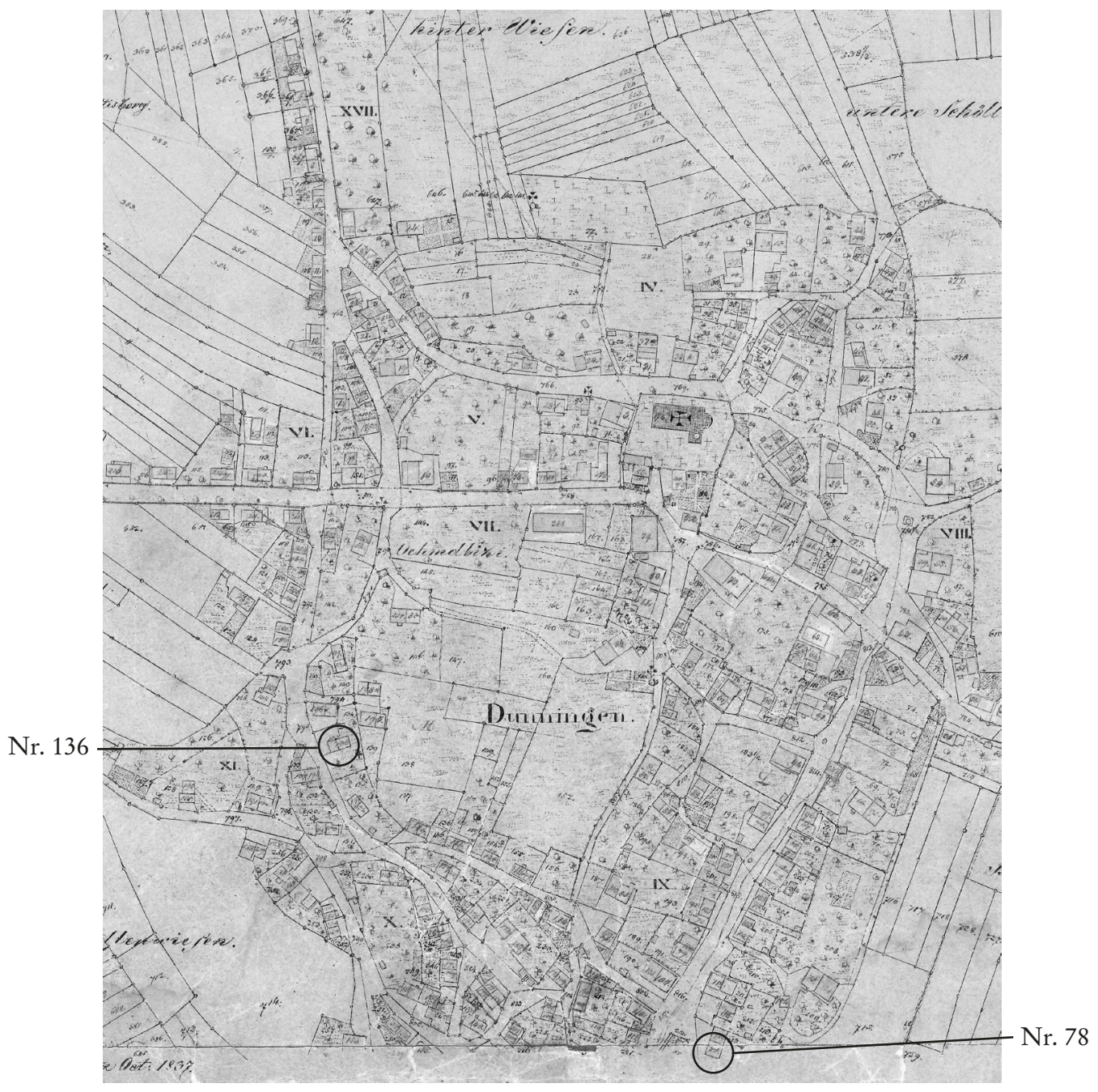

Abb. 2: Urkarte Dunningen 1837 mit dem Geburtshaus von Jacob Mayer mit der Nr. 136 und dem späteren Wohnhaus der Familie Mayer mit der Nr. 78

(Vorlage: Kreisarchiv Rottweil).

Versorgung und damit das Überleben des Nachwuchses sicher zu stellen. Jacob Mayer verliert damit im Alter von acht Jahren seine Mutter und wächst zusammen mit seinen Geschwistern unter der Obhut einer Stiefmutter auf. Aus der zweiten Ehe des Vaters gehen keine Kinder mehr hervor. 


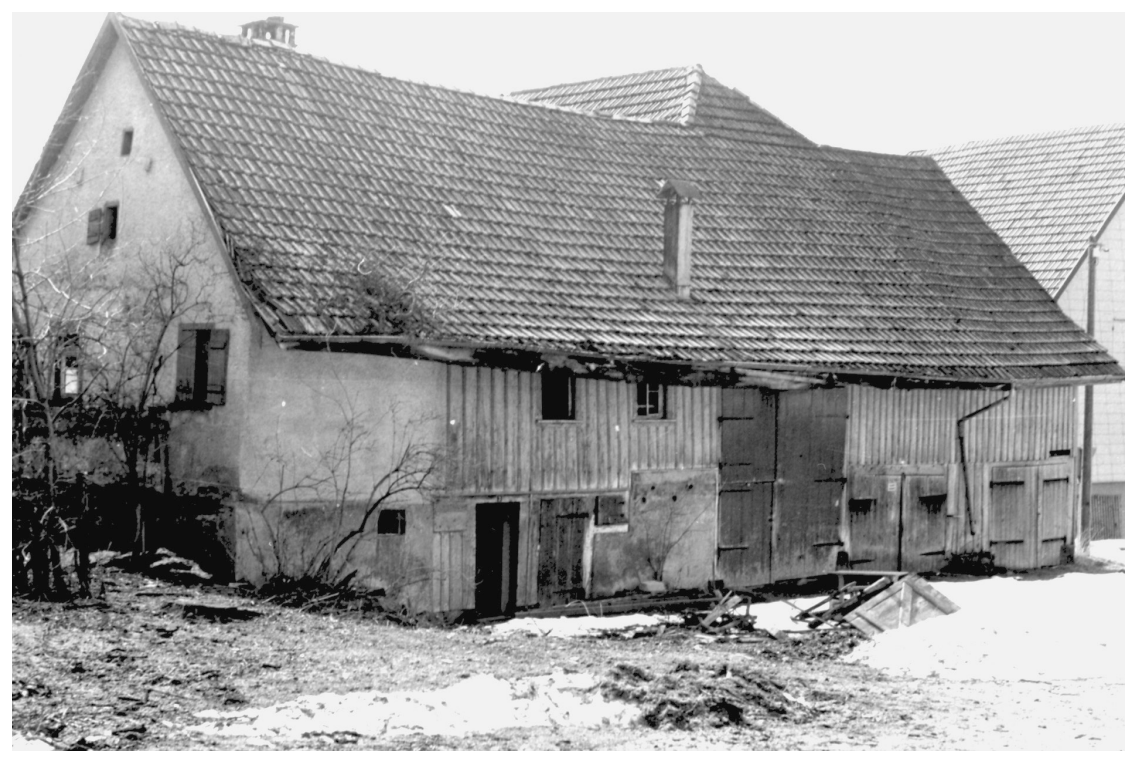

Abb. 3: Das in den 1970er Jahren abgerissene Kleinbauernhaus Nr. 136 an der Ecke Bitzenstraße/Locherhofer Straße in Dunningen - das Geburtshaus von Jacob Mayer (Vorlage: Heimatmuseum Dunningen).

Im Alter von 60 Jahren stirbt am 26. Juni 1833 Adam Mayer und hinterlässt eine Witwe sowie fünf großenteils noch minderjährige Kinder, die es zu versorgen gilt. Dies geschieht durch zwei Kaufverträge nur wenige Wochen nach dem Tod des Vaters: Mit Vereinbarung vom 10. Juli 1833 überlässt die von Pflegern ${ }^{24}$ vertretene Erbengemeinschaft dem ältesten Sohn Josef zum Preis von 2.000 Gulden das Bauernhaus an der Schnurrenstraße nebst Garten, einer Bauernallmend, einem kleinen Viehbestand, den Futter-, Getreide- und Holzvorräten sowie einzeln benannten landwirtschaftlichen Gerätschaften und der erwähnten Wanduhr ${ }^{25}$. Detailliert wird im Vertrag festgehalten, welche Leistungen die Stiefmutter Magdalena Weber neben dem lebenslangen Wohnrecht im Haus von dem Hoferben beanspruchen kann: Ihr stehen die Nutzung der unteren Stube, der Küchenkammer, der unteren Küche sowie ein Drittel des Gartens zu, weiter die Haltung von zwei Stück Vieh im unteren Stall, als jährliches Leibgeding jeweils auf Martini 30 Pfund Salz, 50

${ }^{24}$ Als Pfleger von Josef Mayer werden Lorenz Mauch, als Pfleger seiner vier Geschwister Kaspar Benz und Friedrich Mayer genannt.

${ }^{25}$ Vertrag vom 10.7.1833 über den Verkauf der Verlassenschaft des verstorbenen Adam Mayer, Steuereinbringers, an dessen ältesten Sohn Josef Mayer (Kaufbuch Dunningen, Bd. VI (wie Anm. 22) fol. 76r-81v). 


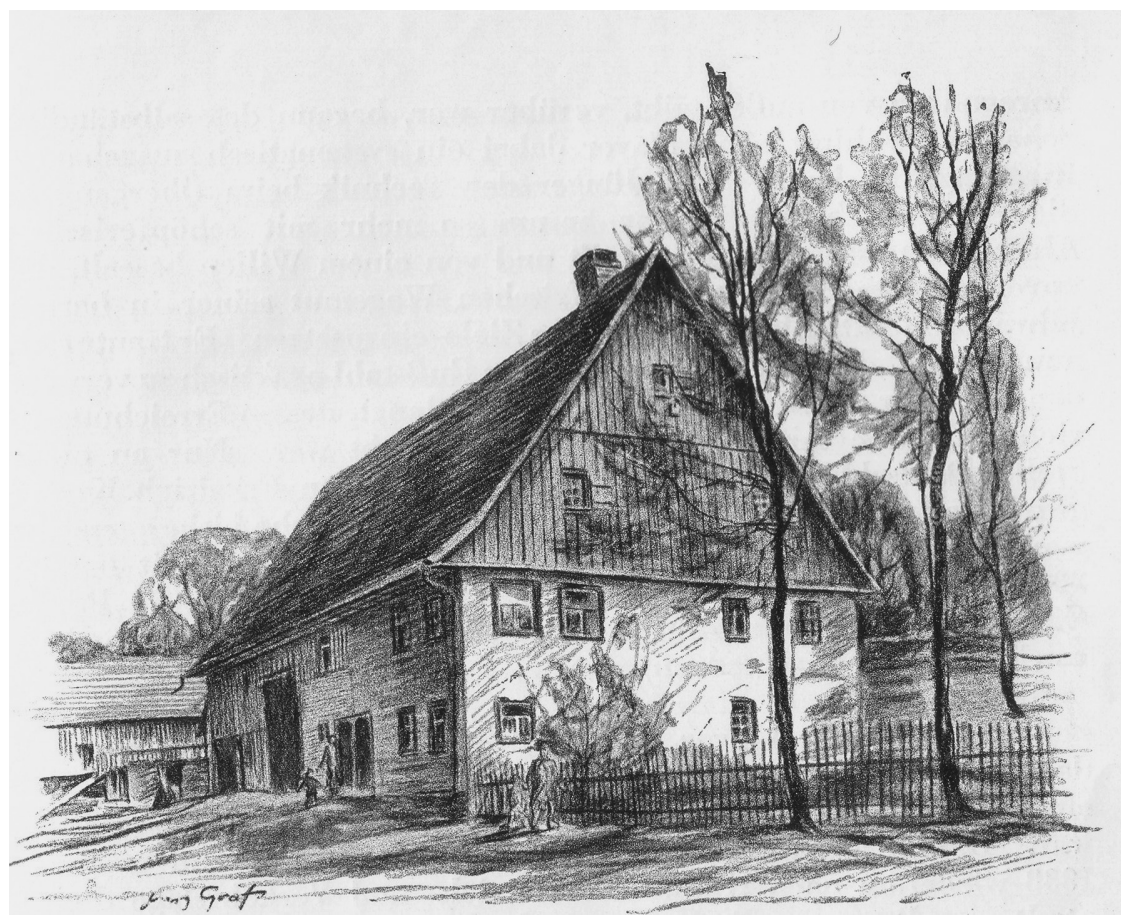

Abb. 4: Bauernhaus Schnurrenstraße 1 in Dunningen, Zeichnung von Franz Graf (Vorlage: Heimatmuseum Dunningen).

Krautköpfe, 15 Simri Grundbieren, d.h. Kartoffeln, 10 Pfund gezupfter Flachs, 1 Klafter Brennholz nebst dem erforderlichen Kochholz, von dem die Mutter allerdings nichts verkaufen darf. Exakt wird bestimmt, welche Felder - 11/2 Jauchert Ackerfelder in allen drei Öschen sowie 1 Jauchert Wiesen - Josef Mayer für seine Stiefmutter alljährlich unentgeltlich zu ackern und zu fahren hat, wobei die erforderlichen Saatfrüchte allerdings Magdalena Weber besorgen muss.

Neben zahlreichen weiteren Bestimmungen wird im Vertrag sogar die freiwillige Verpflichtung des Hauskäufers festgehalten, seiner Stiefmutter jährlich 100 Kübel Dunglache zur Begießung ihres Gartenteils zu geben. Als 21. und letzter Punkt wird im Vertrag dann allerdings auch bestimmt, dass die zu diesem Zeitpunkt 48 Jahre alte Stiefmutter unter keinen Umständen mehr ins Haus heiraten darf, d.h. eine Eheschließung unter dem Dach ihres Stiefsohnes ist ihr ausdrücklich verwehrt. Neben der Stiefmutter räumt der Kaufvertrag auch den jüngeren Geschwistern Maria, Jacob, Fidelis und Sebastian, solange sie ledig sind und sich gut betragen, die Nutzung der sogenannten hinteren Kammer sowie der unteren Stube ein. 
Nach dem Tod der Stiefmutter sollen deren Räume im unteren Stock des Bauernhauses an die Geschwister zur Bewohnung übergehen. Der Kaufvertrag wird von Magdalena Weber mit Handzeichen in Gestalt von drei Kreuzen unterzeichnet ${ }^{26}$.

In einem zweiten Vertrag vom 26. August 1833 wird der gesamte, von Adam Mayer hinterlassene Feldbesitz von 38 Parzellen Äckern und Wiesen zum Preis von zusammen 5.470 Gulden sowie von jährlichen Leibgeding-Leistungen an die Witwe Magdalena Weber verkauft. 21,5 Jauchert gehen dabei an 30 verschiedene Erwerber über, lediglich knapp 7 Jauchert, verteilt auf acht Parzellen, verbleiben dem Hoferben Josef Mayer ${ }^{27}$. Dieser schließt noch im November desselben Jahres die Ehe mit Maria Rottler (1810-1880) und erlangt damit das Bürgerrecht und die volle Verfügung über seinen Besitz ${ }^{28}$. Aus der Verbindung gehen in der Folge insgesamt zehn Kinder hervor, darunter als Letztgeborener der spätere Sattler Josef Mayer junior (1851-1925). Wie zuvor seinem Vater Adam gelingt auch Josef Mayer durch verschiedene Zukäufe von Feldern bis in die 1840er Jahre eine Vergrößerung seines Hofes auf 30,5 Jauchert ${ }^{29}$.

Die Zerstückelung des väterlichen Hofes mit einem Gelderlös von rund 7.500 Gulden dient der materiellen Absicherung der vier jüngeren Geschwister des Hoferben Josef Mayer: Während die Schwester Maria Mayer 1836 den Bauernsohn Dominikus Weber heiratet und in Dunningen eine Familie begründet ${ }^{30}$, streben die drei Brüder Jacob, Fidelis und Sebastian über eine Lehre bzw. ein Studium in Laufbahnen außerhalb des ländlich-bäuerlichen Bereiches und bedürfen über Jahre hinweg der finanziellen Unterstützung aus dem väterlichen Erbe sowie zusätzlich von Josef Mayer. Für die Verhältnisse einer kleinbäuerlichen Familie im 19. Jahrhundert durchaus ungewöhnlich sind dabei insbesondere das Theologie-Studium und die geistliche Laufbahn als Pfarrer von gleich zwei Söhnen. Wie die Korrespondenz von Jacob Mayer mit seinem Bruder Josef aus den 1840er Jahren offenbart, ist das offenkundig in Tübingen absolvierte Studium von Fidelis und Sebastian Mayer ein finanzieller Kraftakt nicht nur für die beiden angehenden Theologen, sondern auch für ihren Dunninger Bruder Josef, der sich beständig von den Studenten und darüber hinaus auch von dem aufstrebenden Erfinder und Unternehmer Jacob Mayer um Unterstützung und Darlehen angegangen sieht ${ }^{31}$. Josef möge doch Fidelis, der zur Befriedigung der nötigsten Bedürfnisse insgesamt 350 Gulden brauche, wenigstens einen Teil des Geldes zuschicken, damit dieser nicht durch eine Verlegenheitspartie am Studieren gehindert werde. Sorgen um sein zu

${ }^{26}$ Ebd., fol. 81v.

27 Vertrag vom 26.8.1833 (ebd., fol. 97v-107r).

${ }^{28}$ Familien-Register I zum Kirchenbuch Dunningen 1808-1909 (wie Anm. 9) S. 320.

${ }^{29}$ Güterbuch Dunningen, Oberamt Dunningen, Bd.VI, fol.2408r-2424v (GemeindeA Dunningen Nr.617).

${ }^{30}$ Familien-Register I zum Kirchenbuch Dunningen 1808-1909 (wie Anm. 9).

31 Schreiben von Jacob Mayer zu Bochum an seinen Bruder Josef Mayer in Dunningen vom 12.5.1844 (wie Anm. 5). 


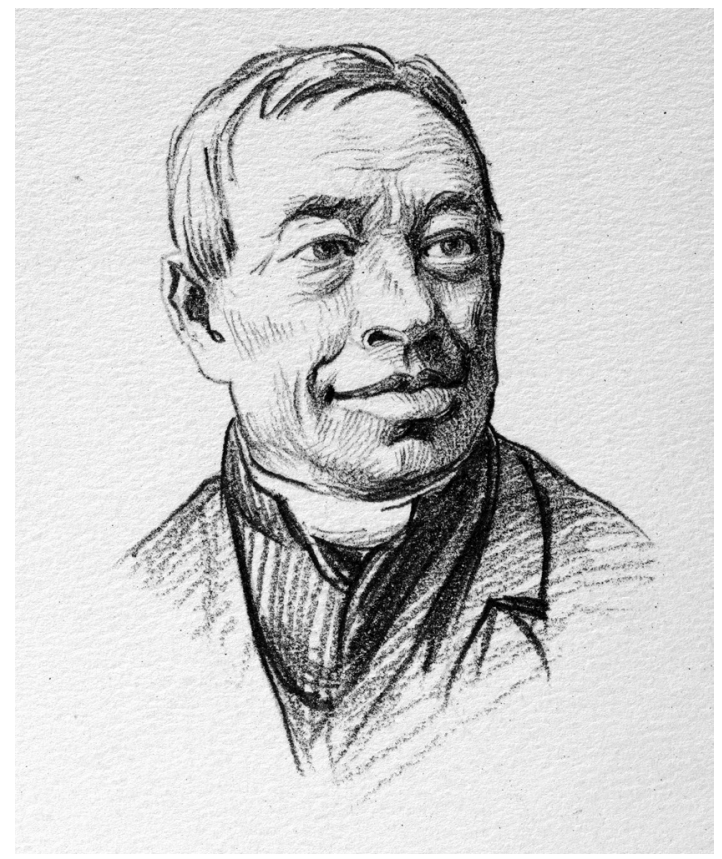

Abb. 5: Pfarrer Fidelis Mayer, Kohlezeichnung, 28,5 x 19,5 cm (Vorlage: Gemeindearchiv Dunningen Nr.1408).

Gunsten seiner Brüder ausgeliehenes Geld müsse er sich nicht machen, denn unter uns drei wird gewis [!] auf der Zeit auch wenigstens [ein Zahlungs-] fäbiger sein, der die erhaltenen Darlehen zurückerstatten könne.

Fidelis Mayer ist nach Studium und Priesterweihe als Gemeindepfarrer in Bayern und hier von 1867 bis 1892 in Söchtenau bei Rosenheim tätig ${ }^{32}$, während Sebastian Mayer in der heimischen Diözese Rottenburg verbleibt und hier u.a. in den oberschwäbischen Pfarreien Schmalegg, Ebersbach und Dürrenwaldstetten seelsorgerlich im Einsatz ist ${ }^{33}$. Die beiden Pfarrherren sind für die in Dunningen verbleibenden Verwandten offenkundig ansehenssteigernd und schlagen sich sogar im Hausnamen der Familie Mayer nieder: Wird der Bauer Josef Mayer zur Unterschei-

32 Schreiben von Dr. Walter Diezinger in Söchtenau an Rektor Julius Wilbs in Dunningen vom 6. Oktober 1993 (Heimatmuseum Dunningen); Julius WiLBS, Die Brüder Jacobs, in: Die Brücke. Dunninger Jahrbuch 1996, S. 48-50.

33 Personalakte Sebastian Maier (DAR Abt. G 1,7,1 Nr. 3603) - nur die Deckblätter der Akte sind erhalten, der Inhalt wurde vernichtet (E. W.); (Priester) Sebastian Maier (!)/Mayer aus Dunningen - Akten des Kath. Kirchenrats (DAR F IV Nr. 880). 
dung von den anderen Mayer-Sippen im Dorf anfänglich mit dem Zusatz Adams nach dem Vornamen seines Vaters näher bezeichnet, so wird in späterer Zeit für ihn wie auch seine Kinder der Hausname Herr(en)maiers gebräuchig ${ }^{34}$ !

\section{Vom Uhrmacherlehrling zum Fabrikbesitzer}

Eine nicht minder ungewöhnliche Berufslaufbahn schlägt der zweitgeborene Sohn Jacob Mayer ein: Nachdem er den Schilderungen seines Neffen Peter Josef Sieber zufolge schon früh einen ausgeprägten Sinn für Mechanik zeigte, wurde er - möglicherweise nach einer ersten Lehre bei einem Mechanikermeister in Rottweil ${ }^{35}$ - in die Ausbildung zu einem Verwandten namens Mauch in Köln gegeben, der sich dort als Uhrmacher niedergelassen und ein Geschäft begründet hatte ${ }^{36}$. Der bereits geschilderten legendenhaften Überlieferung zufolge kam er während seiner Uhrmacherlehre erstmals mit der - noch vergeblichen - Gussstahlfertigung in Berührung. Neben der Lehre erhielt Jacob Mayer durch den Kölner Artilleriemajor Ludwig Bote Unterricht in Mathematik, Physik und Chemie ${ }^{37}$.

Vermutlich nach 1830 geht Jacob Mayer für einige Jahre nach England, wo er den Angaben seines Neffen Peter Josef Sieber zufolge mit riesige $(m)$ Fleiss an seiner Fortbildung arbeitete und die Gebeimnisse der Gussstablfabrikation ergründete und vervollkommnete ${ }^{38}$. Aller Wahrscheinlichkeit nach war er in Fabriken in Sheffield, dem Zentrum der englischen und europäischen Tiegelstahlherstellung, tätig $^{39}$. Auch wenn Jacob Mayer später äußerte, er sei obne genaue Kenntnisse von allem heim (ge)kehrt, ist sich die Forschung weithin einig, dass die in England gewonnenen Erfahrungen und Erkenntnisse ihn „auf seinem Weg zu einer eigenen Tiegelstahlherstellung gewiss vorangebracht" haben ${ }^{40}$.

Legendenhaft und schwerlich zu verifizieren ist die bereits erwähnte Geschichte, wonach Mayer in England das Vertrauen seines Fabrikherrn gewonnen und sich damit zugleich die Feindschaft seiner Arbeitskollegen zugezogen habe, angeblich unter Lebensgefahr ihren Nachstellungen entfliehen und mit knapper Not ein ge-

${ }^{34}$ Als Beispiele Register zum Güterbuch Dunningen 1808ff. (GemeindeA Dunningen Nr. 627); Index zum Güterbuch Dunningen (GemeindeA Dunningen Nr. 625); Güterbuch Dunningen, Bd.VI, fol.2408r (wie Anm. 29); Kaufbücher Dunningen, Bde. XX 1853-1855 (GemeindeA Dunningen Nr.778) und XXII 1855-1858 (Nr.780). Im Güterbuch, Bd. 34, 1877-1880 (Nr. 792) findet sich Josef Mayer junior in Eintragungen vom 15.8.1877, 26.10.1877 und 30.1.1878 mit dem Zusatz Herrenmaiers (fol. 12v, 49r, 82v).

35 Die Rottweiler Lehre wird von Reinert (wie Anm. 2) S.341, ohne weitere Quellenangabe erwähnt.

${ }^{36}$ Schreiben von Peter Josef Sieber an Josef Mayer junior vom 28.10.1904 (wie Anm. 6).

37 Bertram (wie Anm. 2) S. 4; Reinert (wie Anm. 2) S. 341.

${ }^{38}$ Schreiben von Peter Josef Sieber an Josef Mayer junior vom 28.10.1904 (wie Anm. 6).

39 Bertram (wie Anm. 2) S. 4; Rudzinsky (wie Anm. 2) S. 29.

40 Rudzinsky (wie Anm.2) S. 29. 


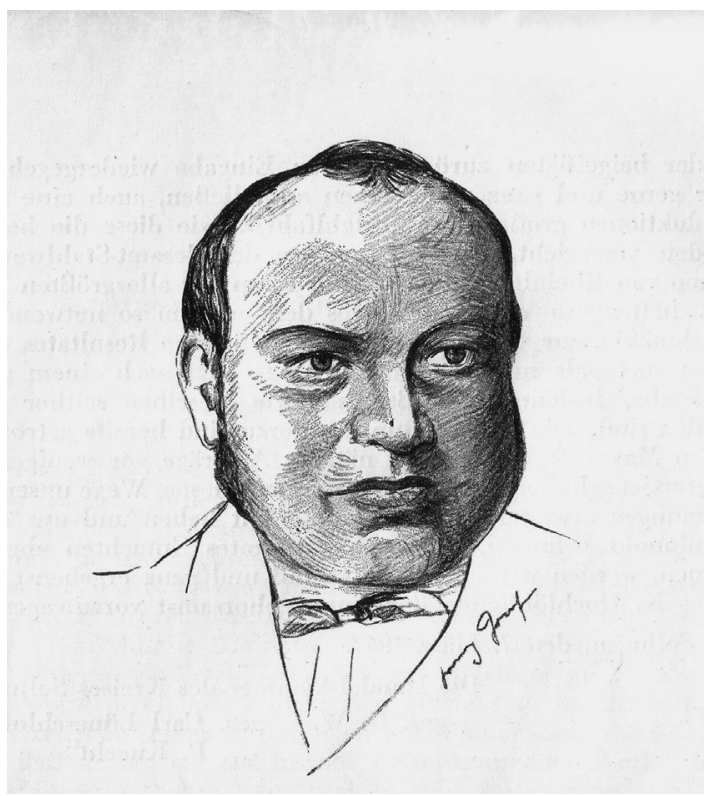

Abb. 6: Jacob Mayer in jungen Jahren, Kohlezeichnung von Franz Graf, 28,5 x $19 \mathrm{~cm}$ (Vorlage: Gemeindearchiv Dunningen Nr. 1408).

rade im Hafen auslaufendes Schiff erreichen konnte, wo er sodann als Schiffsschlosser tätig war und nach mancherlei Fahrten schließlich wieder wohlbehalten die Heimat erreichte ${ }^{41}$. Der wahre Kern der Geschichte könnte in der „Industriespionage“ liegen, die Jacob Mayer wie andere Kontinentaleuropäer, darunter auch sein späterer Kompagnon, der Dürener Fabrikant Eberhard Hoesch, im technisch und industriell fortgeschrittenen England wohl in der Tat betrieben hat ${ }^{42}$.

Die persönlichen und fachlichen Verbindungen Mayers nach England scheinen jedenfalls durch diesen dramatischen Vorfall, wenn es ihn denn gegeben hat, keineswegs gravierend gestört worden zu sein: Einem Brief vom September 1837 an seinen Bruder Josef in Dunningen zufolge will Jacob Mayer nochmals für ein halbes Jahr nach England reisen ${ }^{43}$, und als er im Frühjahr 1861 im Zerwürfnis mit Generaldirektor Louis Baare zum wiederholten Mal den Bochumer Verein für Bergbau und Gussstahlfabrikation verlassen will, hat der englische Lizenzpartner

${ }^{41}$ Bertram (wie Anm. 2) S. 4, erzählt die Geschichte als Erster, ohne allerdings Quellenbelege zu nennen.

42 Ogger (wie Anm. 2) S. 44.

${ }^{43}$ Schreiben von Jacob Mayer zu Köln an seinen Bruder Josef Mayer in Dunningen vom 25.9.1837 (wie Anm. 5). 
Naylor, Vickers \& Co. in Sheffield Interesse an einem Firmeneintritt des mittlerweile europaweit berühmten Erfinders des Stahlformgusses ${ }^{44}$.

Nach seiner Rückkehr nach Deutschland hält sich Jacob Mayer wohl 1836 nochmals für einige Zeit im Heimatdorf Dunningen auf, wo er angeblich im mittlerweile seinem Bruder Josef gehörenden Elternhaus an der Schnurrenstraße erste Versuche zur Herstellung von Gussstahl unter Verwendung von Holzkohle und Tiegeln durchführte ${ }^{45}$. Die Annahme, dass er die benötigten feuerfesten Tiegel in der seit 1820 in Schramberg bestehenden Steingutfabrikation gewonnen habe ${ }^{46}$, ist reine Spekulation. Eindeutig belegt ist dann, wohl 1837, der Umzug und der erste Anlauf zu einer eigenen Gussstahlfabrik in Köln - aus der in England wie in Dunningen gewonnenen Einsicht heraus, dass eine erfolgversprechende Gussstahlherstellung nur mit Steinkohle möglich war.

Den Schilderungen des Neffen Peter Josef Sieber zufolge war die Rückkehr in das Mayer von seiner Uhrmacherlehre noch gut bekannte Köln maßgeblich von der zentralen Handels- und Verkehrslage der Domstadt sowie der Nähe zu den Aachener Kohlegruben bestimmt ${ }^{47}$. Im heutigen Kölner Stadtteil Nippes erwirbt er auf dem Ziegeleigelände seines späteren Schwagers Jacob Volk einen Platz, wo er ein erstes kleines Werk errichtet, das ihm in der Folgezeit vor allem als Laboratorium für seine weiteren praktischen Versuche zur Gussstahlherstellung dient ${ }^{48}$. Schon jetzt in diesem frühen Stadium seiner unternehmerischen Tätigkeit sind das fehlende Betriebskapital und die Bemühungen um Kapitalbeschaffung die große Sorge von Jacob Mayer, die ihn in der Folge in den eineinhalb Jahrzehnten als selbstständiger Fabrikant nicht mehr loslässt und letztlich zu seinem Scheitern als Unternehmer führen sollte.

In den Briefen an seinen Bruder Josef Mayer aus den Jahren 1837 bis $1844 \mathrm{zu}$ nächst aus Köln und später aus Bochum sind die Kapitalbeschaffung und die Gewährung von Darlehen durch den Bruder das alles überragende Thema ${ }^{49}$. Seine ersten unternehmerischen Schritte finanziert Jacob Mayer vor allem mit Kapital aus dem Heimatort Dunningen - in Gestalt seines aus dem Verkauf des väterlichen

${ }_{44}$ Rudzinsky (wie Anm. 2) S. 85.

45 Auch diese Episode wird erstmals von Bertram (wie Anm. 2) S. 5, erwähnt und in den nachfolgenden biografischen Darstellungen übernommen (vgl. ReinerT (wie Anm.2) S.342f.; Ogger (wie Anm. 2) S.44; Rudzinsky (wie Anm.2) S.44, 184). Bei Sieber (wie Anm. 6) findet sich kein Hinweis auf die Dunninger Gussstahl-Experimente. Im Gefolge der „Wiederentdeckung" des Erfinders und Firmengründers Jacob Mayer durch den Bochumer Verein in den 1930er Jahren wird der Kastanienholztisch aus dem Dunninger Elternhaus, wo angeblich die ersten Tiegel standen, als „kostbares Erinnerungsstück“ in das Werksarchiv in Bochum überführt (Bertram (wie Anm. 2) S.5).

46 Reinert (wie Anm. 2) S. 343.

47 Schreiben von Peter Josef Sieber an Josef Mayer junior vom 28.10 .1904 (wie Anm. 6).

48 Reinert (wie Anm. 2) S. 343.

${ }^{49}$ Schreiben von Jacob Mayer aus Köln bzw. Bochum an seinen Bruder Josef Mayer in Dunningen zwischen 1837 und 1844 (wie Anm. 5). 
Hofes gewonnenen und von einem Pfleger verwalteten Erbes sowie aus schlussendlich bis 1839 immerhin 550 Gulden zusätzlicher Darlehen des Bruders und Kleinbauern Josef Mayer $^{50}$. In seinen Briefen bemüht sich Jacob Mayer stets aufs Neue, den offenkundig zögerlichen älteren Bruder von der Sinnhaftigkeit und Risikolosigkeit immer neuer Kredite für seine unternehmerischen Investitionen zu überzeugen. Als letzte Sicherheit benennt er im Januar 1839 sein Stablgeheimnis, also sein mittlerweile erlangtes technisches Wissen um die Gussstahlherstellung, das er notfalls für mehrere tausend Taler verkaufen und damit alle seine Gläubiger befriedigen könnte ${ }^{51}$.

In den Briefen nach Dunningen sind der Fabrikaufbau und die zweimal über Suchanzeigen laufende Gewinnung finanzkräftiger Gesellschafter zunächst 1839 in Nippes und sodann 1842 in Köln und nachfolgend in Bochum dokumentiert: Im Februar 1839 schickt er seinem Bruder eine Anzeige zu, in der Jacob Mayer einen Theilnehmer mit der Bereitschaft zur Investition von 12.000 Talern sucht, von denen ihm für das Geheimnis die Hälfte zufallen soll. Für die Bereisung der insgesamt 13 - und letztlich 16 - Bewerber, darunter Leuten, die hunderttausende von Talern reich seien, um den brävsten herauszusuchen und sich nach ihrem Charakter zu erkundigen, erbittet er bei seinem Bruder einen weiteren Kredit von 350 Gulden $^{52}$. Falls Josef nicht allein helfen wolle, so solle er seinen Schwager Dominikus Weber, also den Ehemann der gemeinsamen Schwester Maria - den Ururgroßvater des Verfassers dieses Beitrags -, zur Hand nehmen.

Drei Jahre später, Ende Dezember 1842, teilt Jacob Mayer letztmals aus Köln seinem Bruder mit, dass er sich mit einem neuen Theilnehmer verbunden habe und beide gemeinsam, sobald dies die Witterung erlaube, eine neue Fabrik bauen wollten ${ }^{53}$. Um sein Geschäft aber betreiben zu können, müsse er preußischer Untertan und Bürger werden und daher beim Oberamt wohl in Rottweil um seine Entlassung aus der württembergischen Staatsangehörigkeit nachsuchen, wofür er von seinem Bruder die Leistung einer jährlichen Bürgschaft erbittet. Im letzten erhaltenen Brief an den Bruder in Dunningen vom Mai 1844 berichtet Jacob Mayer nunmehr aus Bochum, dass die neue Fabrik fast fertig gestellt sei und man noch im laufenden Jahr das Geschäft förmlich eröffnen werde ${ }^{54}$.

Die Korrespondenz Jacob Mayers mit seinem Bruder zeugt von einer engen Verbundenheit der fünf Geschwister und auch zur Stiefmutter Magdalena Weber mit Erkundigungen nach dem Befinden der anderen sowie der regelmäßigen Übermitt-

50 Schreiben von Jacob Mayer an Josef Mayer vom 5.4.1839 (wie Anm. 5) mit beigefügtem Schuldschein über am 15. März 1839 leihweise in bar empfangene 550 Gulden.

${ }^{51}$ Schreiben von Jacob Mayer an Josef Mayer vom 20.1.1839 (wie Anm. 5).

52 Schreiben von Jacob Mayer an Josef Mayer vom 28.2.1839 (wie Anm. 5). Der Hinweis auf 16 Interessenten findet sich im Schreiben vom 13.3.1839 (wie Anm. 5).

${ }^{53}$ Schreiben von Jacob Mayer an Josef Mayer vom 28.12.1842 (wie Anm. 5). Als Adresse in Köln nennt Jacob Mayer Eigelstein Nr. 116.

${ }^{54}$ Schreiben von Jacob Mayer an Josef Mayer vom 12.5.1844 (wie Anm. 5). 
lung von Grüßen. Wenn Jacob Mayer seinem Bruder 1844 Ratschläge für eine lukrativere Geldanlage gibt, so entbehrt dies angesichts der ständigen Finanzsorgen des Erfinders allerdings nicht einer gewissen Komik ${ }^{55}$. Auch von Jacob Mayers bahnbrechenden Erfindungen sollen die Dunninger Angehörigen einen gewissen Nutzen haben, wenn der Unternehmer 1840 seinem Bruder ein vortrefflich schneidendes Rasiermesser aus selbst gemachtem Stahl in Aussicht stell ${ }^{56}$. Alsdann kannst Du Deine Murksmesser alle in Rauch hängen.

Die in der Korrespondenz erwähnte zweimalige Suche nach Geschäftspartnern sowie die gleichfalls geschilderten Fabrikbauten zunächst in Nippes und sodann in Bochum markieren die beiden wesentlichen unternehmerischen Weichenstellungen im Leben von Jacob Mayer. Aus der 1839 angekündigten Ermittlung des brärsten unter den angetretenen 16 Interessenten geht der Dürener Eisenfabrikant Eberhard Hoesch erfolgreich hervor, mit dem Jacob eine gemeinsame England-Erfahrung nebst der "Industriespionage“ in dortigen Puddelstahlwerken verbindet ${ }^{57}$. Mit Hoesch, der in Lendersdorf bereits ein florierendes Hüttenwerk betrieb, wird 1839 ein Gesellschaftervertrag mit der Begründung der Gussstahlfabrik Mayer \& Hoesch abgeschlossen, in die der neue Partner dann allerdings nur 8.000 anstelle der vorgesehenen 12.000 Taler einbringt ${ }^{58}$. Die kaufmännische Leitung des Werkes in Nippes, in dem allerdings lediglich etwa ein halbes Dutzend Arbeiter tätig ist, soll bei Hoesch, die technische Verantwortung dagegen bei Jacob Mayer liegen. Langfristig wird zwischen den beiden Partnern ein Neubau der Gussstahlfabrik in Stolberg bei Aachen und damit in größerer Nähe zu den Hoesch-Werken und den Kohlevorräten des Wurmreviers in Aussicht genommen.

Genau über die Verlegung des Betriebs kommt es in der Folge jedoch zum Dissens und nach drei Jahren letztlich zum Bruch zwischen den beiden Gesellschaftern. Jacob Mayer hatte nämlich inzwischen die bessere Eignung der weniger schwefelhaltigen Ruhrkohle für die Gussstahlherstellung erkannt und strebte - der Coaks wegen - einen Umzug der Fabrik nach Bochum und damit auch in die Nähe des seit Jahrhunderten industriereichen bergischen und märkischen Landes als lukrativer Absatzmarkt an. Eberhard Hoesch ist zu einem solchen risikoreichen Investment fern seines Aachener Geschäftsfeldes indessen nicht bereit ${ }^{59}$.

Einen neuen Geschäftspartner, der den Umzug des Betriebs nach Bochum mitzutragen bereit war, findet Jacob Mayer 1842 wiederum über eine Zeitungsannonce in dem aus Magdeburg stammenden Kölner Kaufmann Eduard Kühne. Am 6. Dezember 1842 kommt es zum Abschluss eines Gesellschaftsvertrags und zur

${ }^{55}$ Ebd.

${ }^{56}$ Schreiben von Jacob Mayer an Josef Mayer vom 18.2.1840 (wie Anm. 5).

57 Bertram (wie Anm. 2) S. 6f.; Ogger (wie Anm. 2) S. 44.

58 Rudzinsky (wie Anm. 2) S.30. Bertram (wie Anm. 2) S. 7 datiert den Gesellschaftervertrag mit 1837 offenkundig zwei Jahre zu früh. Die erwähnte Korrespondenz von Jacob Mayer mit seinem Bruder in Dunningen belegt eindeutig seine Partnersuche 1839.

59 Bertram (wie Anm. 2) S. 7; Ogger (wie Anm. 2) S. 45; Rudzinsky (wie Anm. 2) S. 30 f. 


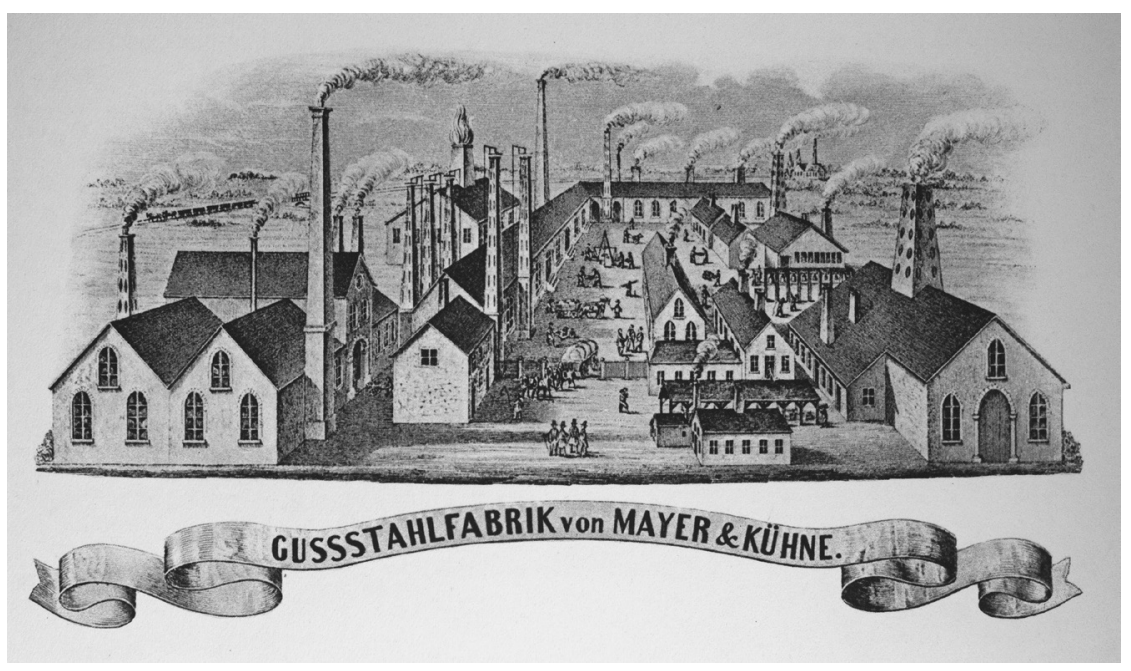

Abb. 7: Gussstahlfabrik Mayer \& Kühne in Bochum, um 1850, Zeichnung (Vorlage: Heimatmuseum Dunningen).

Gründung der Firma Mayer \& Kühne, in welche Jacob Mayer die auf 3.000 Taler geschätzte Nippeser Anlage und Eduard Kühne Investitionsmittel von $15.000 \mathrm{Ta}$ lern einbringen will. Nach dem Erwerb eines Bauplatzes Anfang 1843 westlich der Stadt und unweit des Bergwerks „Präsident“ mit der ersten Tiefbauzeche im Bochumer Raum wird die provisorische Anlage in Nippes demontiert und in dem damals noch recht ländlichen und kaum industrialisierten, rund 4.000 Einwohner zählenden Bochum (Kau-Baukum $\left.{ }^{60}\right)$ wieder aufgebaut ${ }^{61}$. Die Fabrik mit Kühne als kaufmännischem und Mayer als technischem Leiter nimmt 1845 in Bochum ihren Betrieb auf. In der Eifel findet Jacob Mayer in traditionsreichen Standorten der Eisenverhüttung sowohl die benötigten Arbeitskräfte für die angestrebte Produktionsausweitung wie auch hochwertige Tone als geeignete Formmasse für den möglicherweise bereits 1847, spätestens aber 1851 von ihm erfundenen Stahlformguss $^{62}$.

60 Der ländliche Charakter des Kuh-reichen Ackerbürgerstädtchens Bochum wird insbesondere von Peter Josef Sieber in seinem Schreiben an Josef Mayer junior vom 28.10.1904 (wie Anm. 6) betont.

61 Bertram (wie Anm. 2) S. 8f.; Rudzinsky (wie Anm. 2) S. 31 f., 184, 206.

62 Bertram (wie Anm. 2) S. 12 f., 24. 


\section{Erfolgreicher Erfinder und gescheiterter Unternehmer}

Nach einer vor allem der schwierigen wirtschaftlichen Gesamtsituation 1848/ 1849 geschuldeten existenzbedrohenden Krise, als die Gussstahlproduktion stagniert und die Arbeiterzahl zeitweise bis auf weniger als 50 und vorübergehend sogar 15 zurückgeht ${ }^{63}$, erlebt die Bochumer Stahlschmelze dank der revolutionären Erfindungen von Jacob Mayer in den 1850er Jahren einen spektakulären technischen Aufstieg, der in ihren Auftritten bei der Provinzial-Gewerbeausstellung in Düsseldorf 1852 und insbesondere bei der Pariser Weltausstellung 1855 mit der Präsentation großer Gussstahlglocken als erstem mittels Stahlformguss erzeugtem Fabrikat sowie dem viel zitierten Streit mit Krupp um die Qualität des Materials gipfelt und das Unternehmen und zumal den Erfinder Mayer schlagartig international bekannt und berühmt macht ${ }^{64}$. Das Innovative, ja Revolutionäre des von Mayer entwickelten neuen Verfahrens bestand darin, dass die herzustellenden Endfabrikate etwa für den Eisenbahnbedarf, den Maschinenbau, Kanonen oder auch Glocken nicht mehr - wie in England bereits seit dem 18. Jahrhundert und bei Krupp in Essen seit 1815 praktiziert - durch das Durchschmieden des gegossenen Tiegelstahls gewonnen, sondern unmittelbar durch den Guss des flüssigen Stahls in nach Bedarf vorgefertigten, feuerfesten Formen sozusagen nahtlos und final hergestellt werden ${ }^{65}$.

Dem Erfinder gelingt es, die drei für den Erfolg des Stahlformgusses wesentlichen Probleme zu lösen: nämlich einen kontinuierlich dünnflüssigen Stahl bereitzustellen, eine feuerfeste Formmasse zu schaffen, die gleichzeitig auch gasdurchlässig sein muss, um die beim Guss entstehenden Gase entweichen zu lassen und dadurch die Stabilität des sich beim Erkalten zusammenziehenden Stahls zu erhalten $^{66}$. Jacob Mayers Patentgesuch an das preußische Handelsministerium vom 28. Juni 1852 enthält die erste Beschreibung des Stahlformgusses in der Geschichte der Technik ${ }^{67}$.

Ungeachtet ihres spektakulären technischen Aufstiegs in einem in der technikgeschichtlichen Forschung allzu sehr betonten Konkurrenzverhältnis zu Krupp sowie der kontinuierlichen Ausweitung der Produktion in den 1850 er Jahren ist die Firma Mayer \& Kühne, die mit der Serienproduktion des gleichfalls auf Jacob Mayer zurückgehenden nahtlosen Gussstahlscheibenrads zum neben Krupp wichtigsten Zulieferer für die aufstrebende Eisenbahn wird, im Herbst 1853 finanziell am Ende ${ }^{68}$. Die chronische Unterfinanzierung und der Mangel an Eigenkapital, die

63 Rudzinsky (wie Anm. 2) S. 35.

64 Bertram (wie Anm.2) S.26ff.; Reinert (wie Anm.2) S.345f.; Rudzinsky (wie Anm. 2) S. 48.

65 Bertram (wie Anm. 2) S. $1 \mathrm{f}$.

66 Rudzinsky (wie Anm.2) S. 37.

67 Bertram (wie Anm. 2) S. 24.

68 Ogger (wie Anm. 2) S. 46; Rudzinsky (wie Anm. 2) S. 49. 
die unternehmerischen Initiativen von Jacob Mayer von Anfang an und ungeachtet aller mitunter geradezu verzweifelten Bemühungen um Kapitalaufstockungen belastet haben, münden im November 1853 in eine Art Revolution der Gläubiger und Berater des überschuldeten Unternehmens und in die Entmachtung der bisherigen Alleininhaber Jacob Mayer und Eduard Kühne ${ }^{69}$.

An die Stelle der Firma Mayer \& Kühne tritt im Januar 1854 der Bochumer Verein für Bergbau und Gussstahlfabrikation als Aktiengesellschaft mit dem fortan bestimmenden Einfluss von Kölner Kaufleuten und Freiberuflern sowie der Bankhäuser Sal. Oppenheim und Schaffhausenscher Bankverein. Die beiden Eigentümer-Unternehmer werden abgelöst von der Generalversammlung der Aktionäre, dem Verwaltungsrat als eigentlichem Leitungsgremium und letztlich einem bevollmächtigten Generaldirektor als operativer Führungsinstanz ${ }^{70}$. Während Eduard Kühne 1858 endgültig aus dem Verwaltungsrat und dem Unternehmen ausscheidet, bleibt Jacob Mayer seiner Gründung auch unter den neuen Eigentums- und Führungsverhältnissen als wichtiger Aktionär, vor allem aber als Technischer Direktor bis zu seinem Tod 1875 erhalten.

Die weitere Entwicklung des Bochumer Vereins, der sich von einer kleinen Gussstahlfabrik zu einem expansiven vertikalen Konzern und einem der in Deutschland bis zum Ersten Weltkrieg führenden Qualitätsstahlwerke mit einem weitgespannten Tätigkeitsspektrum wandelt ${ }^{71}$, soll hier nur soweit interessieren, wie Jacob Mayer damit in Verbindung steht. Die durchaus ungenügenden, kaufmännisch vielleicht sogar dilettantischen Geschäfts- und Betriebsmethoden von Mayer \& Kühne werden unter der Leitung des neuen Generaldirektors Louis Baare seit 1854 durch eine straffe und leistungsfähige Unternehmensorganisation abgelöst ${ }^{72}$.

Die von dem erfahrenen Kaufmann Baare betriebene forcierte Bürokratisierung und Ökonomisierung des Unternehmens mit zunehmenden Eingriffen auch in die Organisation des von Mayer geleiteten technischen Betriebs führen alsbald zu massiven Spannungen und Konflikten mit dem früheren Fabrikinhaber. Die Höhepunkte der sich über mehrere Jahre hinziehenden Auseinandersetzungen bilden 1858 die Kündigung Baares als Generaldirektor und seine kommissarische Wahrnehmung der Firmenleitung bis 1861 sowie gleich mehrere Entlassungsgesuche Mayers in den Jahren 1859 bis 1861, die der Verwaltungsrat jeweils zurückweist, da man auf seinen technischen Sachverstand nicht verzichten will ${ }^{73}$.

Am Ende des Konflikts steht Ende 1861 etwas überraschend die Einwilligung des in dieser Frage zuvor sturen Jacob Mayer in die von Baare geforderte Delegie-

69 Rudzinsky (wie Anm. 2) S. 32, 37, 39f.; Ogger (wie Anm. 2) S. 46.

70 Rudzinsky (wie Anm. 2) S. 39-47; Bertram (wie Anm. 2) S. 38 f.

${ }^{71}$ Grundlegend zur Firmengeschichte bis zum Ersten Weltkrieg die Studien von Däвritz und Rudzinsky (wie Anm.2).

72 Reinert (wie Anm. 2) S. 347; Rudzinsky (wie Anm. 2) S. 45.

${ }^{73}$ Zum Konflikt zwischen Baare und Mayer vgl. Rudzinsky (wie Anm. 2) S. $83 \mathrm{ff}$. 


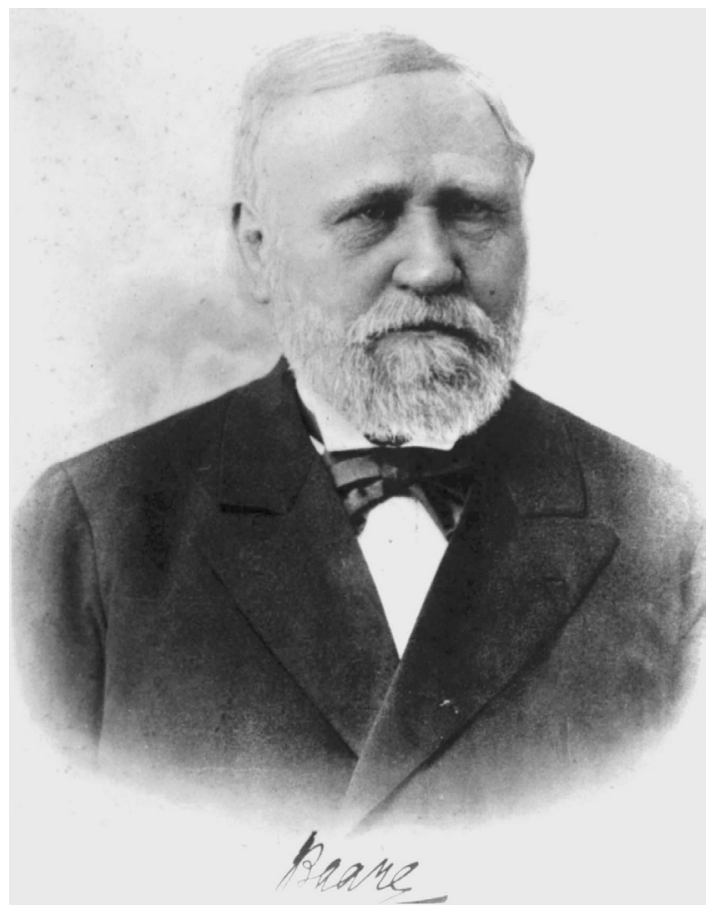

Abb. 8: Generaldirektor Louis Baare (1821-1897) (Vorlage: Stadtarchiv Bochum).

rung der technischen Zuständigkeit auf mehrere Spartenverantwortliche unter der nominell fortbestehenden Gesamtleitung des Technischen Direktors. Mit der vom Verwaltungsrat mit Zustimmung Mayers im April 1862 beschlossenen Neuregelung der technischen Verwaltung ist der Hauptgrund für die Differenzen zwischen ihm und Baare weggefallen und nähern sich die beiden so weit an, dass sich Mayer von der Generalversammlung im September 1862 für die Baare-Partei in den Verwaltungsrat wählen lässt und Baare damit den alten Gegner in die neue Unternehmensleitung als Mitglied seiner Richtung integrieren kann ${ }^{74}$. Der Bochumer Verein steht damit exemplarisch für eine in der gesamten Industrie um sich greifende Tendenz, dass kaufmännisch bzw. juristisch geschulte Manager zum Nachteil der Techniker in den Firmenleitungen die Oberhand gewinnen ${ }^{75}$.

\footnotetext{
74 Ebd., S. 85 f.

75 Ebd., S. 83.
} 


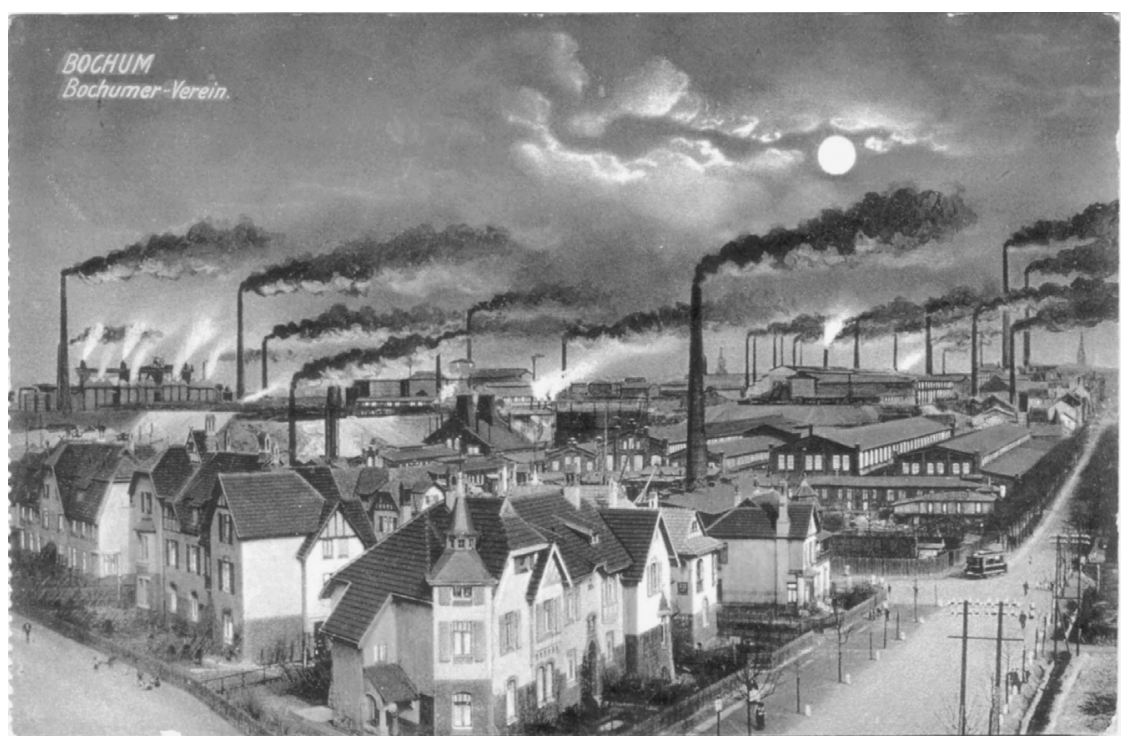

Abb. 9: Bochumer Verein, Bildpostkarte um 1910 (Vorlage: Edwin Ernst Weber).

Auch wenn der Neffe Peter Josef Sieber von einer anhaltenden Entfremdung zwischen den gänzlich gegensätzlichen Persönlichkeiten Baare und Mayer und fortbestehenden Rückzugsplänen seines Onkels berichtet ${ }^{76}$, bleibt die Tatsache, dass Jacob Mayer bis zu seinem Tod 1875 dem Bochumer Verein mit offenbar unvermindertem Einsatz als Technischer Direktor dient und die Einführung weiterer Innovationen wie des Bessemer-Verfahrens 1865 oder des Siemens-Martin-Verfahrens Anfang der 1870er Jahre führend begleitet ${ }^{77}$. In den Anfängen erlebt er noch den Ausbau seines Werks zum Großkonzern mit angegliederten Kohlezechen, Erzgruben und Hochöfen.

Als Mayer am 30. Juli 1875 stirbt, ist aus der 1842 von ihm begründeten kleinen Stahlschmelze mit ein paar Dutzend Arbeitern, einer jährlichen Produktionsleistung von einigen Tausend Zentnern Gussstahl und einem Betriebskapital von wenigen 10.000 Talern ein Großunternehmen mit mehr als 4.500 Beschäftigten, der Erzeugung von 9.000 Tonnen Tiegelgussstahl und einer Bilanz von 27 Millionen Mark geworden ${ }^{78}$. Die Beschäftigten des Bochumer Vereins zählen mit ihren Fami-

76 Schreiben von Peter Josef Sieber an Josef Mayer junior vom 28.10.1904 (wie Anm. 6); Schreiben von Peter Josef Sieber an Karl Schneider vom 20.5.1907 (wie Anm. 6).

77 Bertram (wie Anm. 2) S. $41 \mathrm{f}$.

78 Ebd., S. 42 f.; Neumann zufolge (wie Anm. 8) S. 147 zählte der Bochumer Verein beim Tod Mayers sogar 5.650 Arbeiter und 250 Beamte. 
lien mehr als die Hälfte der damals 28.562 Einwohner Bochums ${ }^{79}$, das der Gründung Jacob Mayers bis zum Ersten Weltkrieg den Aufstieg zur Industriemetropole und Großstadt zu verdanken hat ${ }^{80}$.

Dank der von drei Mitgliedern der Familie Baare in leitender Position über 60 Jahre bestimmten Erinnerungskultur gerät beim Bochumer Verein das Wissen um seinen Firmengründer Jacob Mayer zeitweise in Vergessenheit und wird beim 50-jährigen Bestehen der Aktiengesellschaft 1904 der zur zentralen Bezugsgestalt des Konzerns stilisierte Louis Baare in der Festschrift gar als „Begründer“ des Werks bezeichnet ${ }^{81}$. Von Mayers Witwe Agnes Sieber und kaum minder von seinem Neffen Peter Josef Sieber wird diese postume Missachtung des Firmengründers und seinerzeit größten Technikers Deutschlands als bitterer Undank empfunden, den sie letztlich auf Mayers Persönlichkeit und vor allem Katholizität zurückführen. Wäre er zu den Liberalen oder Freimanern [!] gegangen, dann wäre er auch heute noch verherlicht [!], aber so...? er ist nicht mehr, schreibt Peter Josef Sieber 1904 voller Bitterkeit nach Dunningen ${ }^{82}$. Das von der Witwe Agnes Sieber über dem Grab ihres Mannes auf dem damaligen städtischen Friedhof, dem heutigen Kortumpark, 1877 gestiftete imposante neogotische Grabdenkmal ist mit seiner aufwändigen Ausstattung mit mehreren Heiligenfiguren, einer Büste des Verstorbenen und nicht zuletzt einer von Papst Pius IX. aus der Callixtus-Katakombe in Rom persönlich erhaltenen Reliquie ein Zeugnis der Katholizität Mayers und zugleich ein steinerner Protest gegen sein Vergessen in Betrieb und Öffentlichkeit ${ }^{83}$.

Das Vergessen des Firmengründers und Erfinders Jacob Mayer hat indessen keinen Bestand: Zum 80-jährigen Jubiläum des Bochumer Vereins und aus Anlass der formalen Wiederverselbständigung des 1926 in die Vereinigten Stahlwerke eingegliederten Unternehmens legt der renommierte Wirtschaftshistoriker Walter Däbritz 1934 eine voluminöse Firmengeschichte mit einer Würdigung der Verdienste von Mayer vor. 1935 folgen eine Gedenkplastik für Mayer an der neu erbauten Abnahmezentrale des auf strammem NS-Kurs fahrenden Betriebs ${ }^{84}$ und 1938 zum 125. Geburtstag Mayers eine von Walter Bertram verfasste biografische Studie sowie ein vom Verein Deutscher Ingenieure an Mayers - vermeintlichem Geburtshaus in Dunningen angebrachte Gedenktafel und vollenden die erinnerungsmäßige „Rehabilitierung“ des Erfinders und Firmengründers ${ }^{85}$.

79 Neumann (wie Anm. 8) S. 147.

80 Zum durchaus spannungsreichen Verhältnis zwischen der Stadt Bochum und ihrem führenden Industriebetrieb vgl. Rudzinsky (wie Anm. 2) v. a. S. 222 ff., 269 ff., 407 f.

${ }^{81}$ Ebd., S. 169.

82 Schreiben von Peter Josef Sieber an Josef Mayer junior vom 28.10.1904 (wie Anm. 6).

${ }^{83} \mathrm{Zu}$ Entstehung und Ausstattung des Grabdenkmals vgl. Neumann (wie Anm. 8).

${ }^{84}$ Zur Geschichte des Bochumer Vereins in der NS-Zeit vgl. Gustav-Hermann SEEBold/ Alfred Wortmann, Ein Stahlkonzern im Dritten Reich. Der Bochumer Verein 1927-1945, Wuppertal 1981.

85 Rudzinsky (wie Anm. 2) S. 15, 17; Reinert (wie Anm.2) S. 340, 349; Bertram (wie Anm.2) S. 49. 


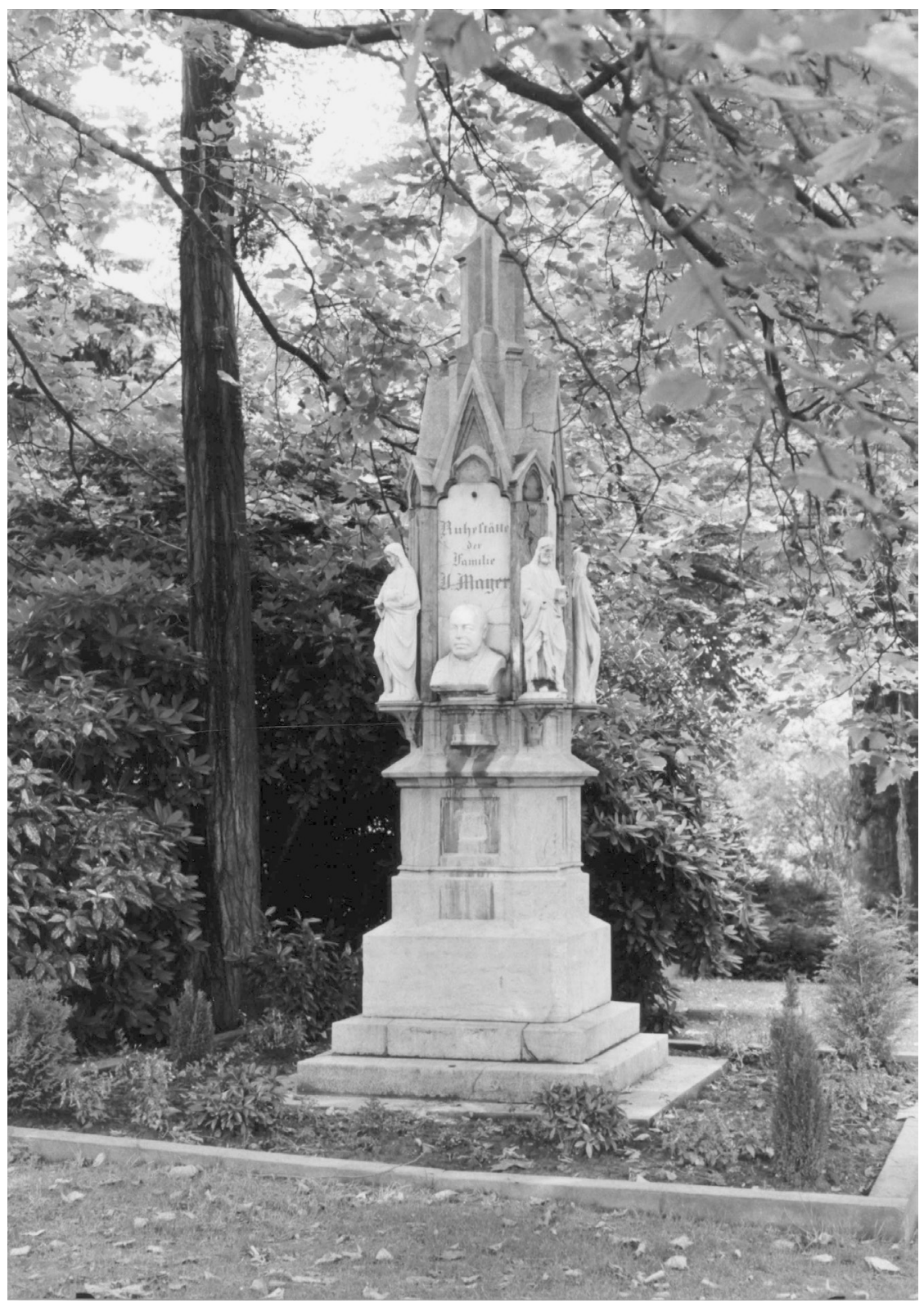

Abb. 10: Grabmal von Jacob Mayer auf dem alten Bochumer Friedhof, dem heutigen Kortumpark, Aufnahme von 1985 (Vorlage: Stadtarchiv Bochum).

Zeitschrift für Württembergische Landesgeschichte 74 (2015), S. 173-200.

(C) Kommission für geschichtliche Landeskunde in Baden-Württemberg und Württembergischer

Geschichts- und Altertumsverein e.V.

ISSN 0044-3786 


\section{Persönlichkeit und Katholizität}

Es bleibt abschließend noch ein Blick auf Persönlichkeit und Charakter von Jacob Mayer zu werfen, die entscheidend von seiner tiefen Frömmigkeit und Katholizität geprägt werden. Die vielen Stunden, die ich in Bochum zubrachte, erinnert sich der Neffe Peter Josef Sieber, waren ernst, sebr ernst. Ein ernstes Haus, ernstes Geschäft, dessen Ernst sich auch auf das Familienleben übertrug. Jacob Mayer habe nur für die Ehre Gottes wirkend fast heiligmäßig gelebt ${ }^{86}$. In der Tat stand Mayer treu [...] zu seiner hl. Kirche ${ }^{87}$ mit einem auch öffentlichen Bekenntnis zu seinem Glauben.

Jacob Mayer ist in seinen Bochumer Jahren einer der bekanntesten Katholiken in der Stadt, der seiner Kirche lange Zeit als Gemeindevertreter dient. So zählt er in den 1860er Jahren zu den Initiatoren für die hürdenreiche und zunächst scheiternde Errichtung einer zweiten katholischen Kirche in der Stadt und engagiert sich nachdrücklich für die Gewährung eines Zuschusses durch den Bochumer Verein, den die Hauptversammlung auf Vorschlag von Louis Baare allerdings auf 1.000 anstelle der von Mayer zunächst beantragten 5.000 Taler reduziert ${ }^{88}$.

Seine besondere Förderung ließ Jacob Mayer der Ansiedlung eines Redemptoristenklosters in Bochum seit 1868 angedeihen. Nachdem der Prior zunächst ohne Erfolg bei Generaldirektor Baare die Schenkung eines vierglockigen Geläuts für den Klosterneubau erbeten hatte, stiftet Jacob Mayer aus eigenen Mitteln die größte Glocke und kann das Kloster die übrigen drei Glocken vom Bochumer Verein mit einem Rabatt von 50 Prozent beziehen ${ }^{89}$. Mayer hält den Patres auch im bald einsetzenden preußischen Kulturkampf die Treue, und als ein Regierungsdekret vom 26. Juni 1873 die Auflösung des Klosters und die Ausweisung der Patres verfügt, begleitet er den Superior demonstrativ in einem Galawagen zum Bahnhof ${ }^{90}$.

Jacob Mayer ist Anfang 1872 auch an der Gründung der Märkischen Vereinsdruckerei beteiligt, die seit dem nachfolgenden Sommer die „Westfälische Volkszeitung“ als streitbares und von den preußischen Behörden wiederholt für „Pressevergehen" bestraftes Organ des auch in Bochum entstehenden katholischen Milieus herausgibt ${ }^{91}$.

Die ihm von Papst Pius IX. persönlich geschenkte Reliquie dürfte ebenso eine Belohnung und Auszeichnung für Mayers katholische Standhaftigkeit und öffentliches Bekenntnis zu seiner Kirche gewesen sein wie der ihm 1871 verliehene Gregorius-Orden als der höchsten vatikanischen Auszeichnung. Jacob Mayer nimmt

\footnotetext{
86 Schreiben von Peter Josef Sieber an Karl Schneider vom 20.5.1907 (wie Anm. 6).

87 Schreiben von Peter Josef Sieber an Josef Mayer junior vom 28.10.1904 (wie Anm. 6).

88 Rudzinsky (wie Anm. 2) S. 401.

89 Ebd., S. 408.

90 Neumann (wie Anm. 8) S. 149.

91 Rudzinsky (wie Anm. 2) S. 356.
} 
in der Folge im Ornat eines St. Gregorius-Ritters an der alljährlichen Fronleichnams-Prozession in Bochum teil ${ }^{92}$.

Jacob Mayer ist offenkundig gut vernetzt im katholischen Milieu des Rheinlandes und Westfalens und steht mit dem Kölner Dombaumeister Vincenz Statz ebenso in Verbindung wie mit Adolph Kolping ${ }^{93}$. Eine enge Beziehung pflegt Jacob Mayer auch zu seinen beiden Priester-Brüdern in Bayern und in Oberschwaben, von denen der Jüngere, Sebastian Mayer, als ausgesprochen streitbarer ultramontaner Priester mehrfach mit Staatsbehörden, Gerichten und Gemeindemitgliedern in Konflikt gerät und 1874 wegen der öffentlichen Beleidigung von Kaiser Wilhelm I. als Schnapslump zu sechs Monaten Festungshaft verurteilt wird ${ }^{94}$. Sebastian und Fidelis Mayer reisen nicht nur zur Beerdigung ihres Bruders nach Bochum an, sondern bleiben in der Folge in engem Kontakt mit seiner Witwe und beraten sie bei der Festlegung des Bildprogramms für das Grabmal von Jacob Mayer ${ }^{95}$.

Bekannt sind Jacob Mayer und seine Frau Agnes auch für großzügige Almosenspenden und Gaben für kirchliche Zwecke. So hat es durchaus seine Logik, dass das kinderlose Ehepaar Jacob und Agnes Mayer in seinem Testament vom Juli 1873 den Bischöflichen Stuhl in Paderborn oder ersatzweise weitere katholische Bistümer in Deutschland, Belgien, Frankreich und England als Universalerben mit der Auflage einsetzt, den Nachlass für die Zwecke des Bonifatius- sowie des Xaverius-Missionsvereins zu verwenden ${ }^{96}$. Die Behörden ermitteln nach dem Tod von Jacob Mayer 1875 und gleichermaßen nach dem Ableben seiner Witwe Agnes Sieber 1900 erbberechtigte Angehörige der Verstorbenen und deren Bedürftigkeit, so dass letztlich wohl doch nicht der gesamte Nachlass der Kirche zugefallen sein dürfte ${ }^{97}$.

Jacob Mayer ist am Ende seines Lebens ein reicher Mann mit einem - wohl jährlichen - Direktorengehalt von 15.000 $\mathrm{Mark}^{98}$, Tantiemen für seine Erfindungen, Dividenden der - 450 bereits im Jahr 1862 von ihm und seiner Frau gehaltenen -

92 Neumann (wie Anm. 8) S. $147 \mathrm{f}$.

93 Ebd., S. 151.

94 Kirchenrats-Akte Sebastian Mayer (wie Anm. 33); Neckarbote Rottenburg-Tübingen vom 27.3.1875, S. 144; Schreiben von Pfarrer Dr. Laupheimer in Ebersbach-Musbach an Rektor Julius Wilbs in Dunningen vom 11. November 1993 mit Auszügen aus der Pfarrchronik von Ebersbach, verfasst von Pfarrer Fischinger zu dessen Vorvorgänger Pfarrer Sebastian Mayer (Heimatmuseum Dunningen).

95 Neumann (wie Anm. 8) S. 154. Neumann zufolge sind die beiden Brüder als Geistliche in Rom tätig, was definitiv nicht den Tatsachen entspricht.

96 Testament von Jacob Mayer und Agnes Mayer geborene Sieber vom 2. Juli 1873 zu Bochum, Abschrift (Historisches Archiv Krupp WA 80/1421; Kopie Heimatmuseum Dunningen).

97 Nachlassregelung Verstorbener 1874-1906, enthält u.a. Fabrikdirektor Jacob Mayer und Agnes Mayer, Witwe des Fabrikdirektor J. Mayer (Stadtarchiv Bochum B 324, Film Nr.12BOA2004000004).

98 Schreiben von Peter Josef Sieber an Jacob Mayer junior vom 28.10.1904 (wie Anm. 6). 
Aktien des Bochumer Vereins ${ }^{99}$ sowie einem Privatvermögen von angeblich 800.000 Mark ${ }^{100}$. Seiner Witwe Agnes Sieber ist es offenbar kurz vor ihrem Tod möglich, aus ihren angesparten Einkünften 105.000 Mark für die Kinder ihrer verstorbenen Geschwister abzuzweigen und darüber hinaus noch eine Familien-Studienstiftung mit einem Kapital von 60.000 Mark zu begründen; 73.000 Mark gehen durch staatliche Verfügung aus dem Nachlass des Ehepaares nach 1900 an die zahlreichen Nachfahren (Dessendenz) von Jacob Mayers Geschwistern in Dunningen $^{101}$.

Den Schilderungen ihres Neffen Peter Josef Sieber zufolge führten Jacob und Agnes Mayer, die nach zwölfjähriger Bekanntschaft 1854 im Alter von 41 und 42 Jahren die Ehe geschlossen hatten, ein einfaches und eher schlichtes Familienleben und bewohnten lange Zeit ohne Dienstmädchen die erste Etage eines bescheidenen Hauses $^{102}$. Sie teilten sich, so berichtet Sieber weiter, in erster Linie Arbeit und religiöse Pflichten, waren sparsam in ihren Ausgaben, spendeten aber reichlich Almosen. Die einzige Erholung habe für Jacob Mayer darin bestanden, alljährlich einige Wochen im Sommer in einem nahe gelegenen Badeort zu verbringen. Erst nach langen Jahren wurde für 28.000 Taler ein etwas repräsentativeres neues Haus gekauft, in dessen Saal Sieber zufolge dann auch wiederholt Festlichkeiten abgehalten wurden. Nach dem Tod der Witwe wurde das Mayersche Anwesen von der Stadt Bochum für angeblich 400.000 Mark zur Erweiterung des benachbarten Rathauses erworben ${ }^{103}$.

Einige Verwandte aus Dunningen folgen Jacob Mayer offenkundig nach Bochum: Als nach dem Tod des Fabrikdirektors 1875 dessen erbberechtigte Verwandte ermittelt werden, werden neben den beiden Pfarrer-Brüdern Fidelis und Sebastian Mayer sowie den Kindern des mittlerweile verstorbenen Bruders Josef sowie der Schwester Maria in Dunningen auch der 74 Jahre alte Fabrikarbeiter Andreas Mauch aus Bochum, ein Vetter von Jacob Mayer, der Fabrikarbeiter Gottfried Mauch, ein Neffe wiederum von Andreas Mauch, und schließlich noch die gleichfalls verwandte Maria Mauch aktenkundig, die insgesamt 27 Jahre als Dienstmagd zunächst für das Ehepaar Mayer und anschließend bis zu deren Tod 1900 für die Witwe Agnes Sieber tätig ist ${ }^{104}$.

Jacob Mayer erscheint im Rückblick als ebenso kantige wie ambivalente Persönlichkeit: Da ist zum einen der technikbesessene Erfinder, der als Autodidakt und

99 Rudzinsky (wie Anm. 2) S. 96. Das Ehepaar Mayer ist damit noch vor Louis Baare mit 279 Aktien gemeinsam der größte Einzelaktionär des Bochumer Vereins.

100 Neumann (wie Anm. 8) S. 154.

101 Nachlassregelung Verstorbener 1874-1906 (wie Anm. 97) mit dem Protokoll der Vernehmung von Peter Josef Sieber am 31.7.1900 in Trier; Erbschaftssache Jacob und Fidelis Mayer 1892-1900 (Nachlass Ernst Weber senior im Besitz des Verfassers dieser Schrift). Tatsächlich nachweisen lässt sich die Auszahlung von 27.300 Mark.

102 Schreiben von Peter Josef Sieber an Jacob Mayer junior vom 28.10.1904 (wie Anm. 6).

103 Ebd.; Rudzinsky (wie Anm. 2) S. 417.

104 Nachlassregelung Verstorbener 1874-1906 (wie Anm. 97). 


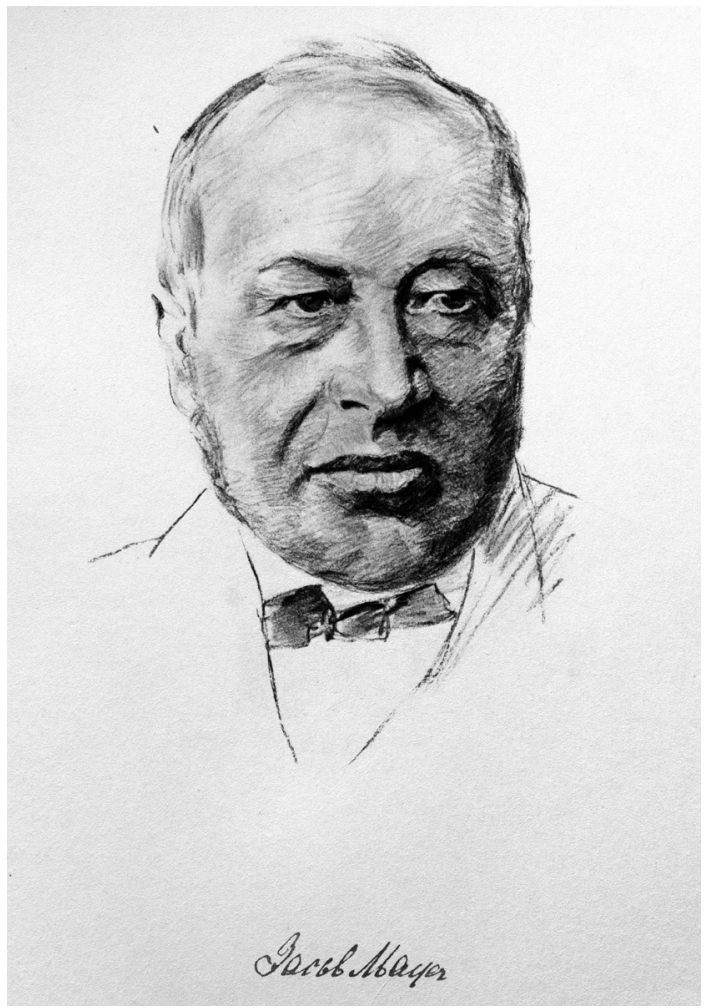

Abb. 11: Jacob Mayer in älteren Jahren, Zeichnung (Vorlage: Gemeindearchiv Dunningen Nr.1408).

ohne solide theoretische Vorbildung durch Zähigkeit und endlose Versuche zu einem der führenden Metallurgen seiner Zeit wird und einen wegweisenden Beitrag für die europäische Technik- und Industriegeschichte leistet ${ }^{105}$. Der spätere Bundespräsident Theodor Heuss sieht im Vergleich zu dem in den Führungstalenten überlegenen Konkurrenten Krupp in Mayer „das stärkere unmittelbar technische Ingenium“, in dem „ein unmittelbares, irrationales Empfinden für den Stoff gesteckt“ haben müsse. In der „eigentümlichsten Weise“ war Jacob Mayer „werkbesessen“ und verkehrte angeblich nur mit den alten, ihm vertrauten Arbeitern. Wenn er in den Fabrikhallen mit einer Laterne nächtliche Kontrollgänge macht, um die Leistung, Sorgsamkeit und Sparsamkeit zu überprüfen, erkennt Heuss „in

105 Vgl. Bertram (wie Anm. 2) S. 44. 
ihm etwas von dem sorgsamen Bauern, der in der Nachtstunde den Weg durch Stall und Scheuer macht, um zu sehen, ob alles in Ordnung sei“"106.

Die andere, dunklere Seite in Jacob Mayers Charakter ist seine „fast unzugängliche Natur" und seine schwierige, mitunter geradezu schroffe Eigenwilligkeit ${ }^{107}$. Der fromme, arbeitsame und in gewissem Sinne pedantische Mann blieb trotz aller hohen Auszeichnungen für seine bahnbrechenden Erfindungen ${ }^{108}$ zurückhaltend, ja eigenbrötlerisch, alles Glamoureuse, Renommierende, Blendende blieb ihm trotz aller Erfolge fremd. Den persönlichen Anschluss an die sich in dieser Zeit im Rheinland und im Ruhrgebiet bildende industrielle Führungsschicht hat er nicht gefunden und wohl auch nicht gesucht ${ }^{109}$. Seine Bezugswelt, in der er sich aufgehoben und heimisch fühlte, waren neben der Technik die Familie sowie die katholische Kirche.

Man tut dem Dunninger Landsmann und genialen Erfinder und „Technikfreak“, wie man heutzutage sagen würde, aber wohl nicht unrecht, wenn man auch auf sein Scheitern als Unternehmer und letztlich auf sein kaufmännisches und ökonomisches Unvermögen hinweist. Jacob Mayer war in der Tat „kein guter Verkäufer und ein miserabler Kaufmann" ${ }^{110}$, der „von Geschäften wenig verstand und sich

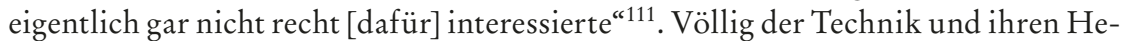
rausforderungen verfallen, tat er sich schwer im Umgang mit Geschäftspartnern, Kunden und zumal seinem nominellen Vorgesetzten Louis Baare, auch wenn die Differenzen zwischen den beiden nicht - wie in der älteren Literatur beschrieben - vorrangig auf „die Unterschiede in Persönlichkeit und Charakter zwischen dem katholischen Stahltechniker aus Württemberg und dem protestantischen Kaufmann aus Westfalen“ zurückgeführt werden dürfen, sondern komplexeren, strukturellen Hintergründen zumindest mitgeschuldet waren ${ }^{112}$. Es bleibt mithin das Bild einer widersprüchlichen und gleichwohl beeindruckenden Persönlichkeit, die auch 200 Jahre nach ihrer Geburt noch Aufmerksamkeit verdient.

106 Heuss (wie Anm. 2) S. 161.

107 Bertram (wie Anm. 2) S. 46; Rudzinsky (wie Anm. 2) S. 83.

108 Jacob Mayer erhielt von Kaiser Napoleon III. bei der Pariser Weltausstellung 1855 das Kreuz der Ehrenlegion verliehen, ein württembergischer Orden sowie der preußische rote Adlerorden folgen nach (Schreiben von Peter Josef Sieber an Josef Mayer junior vom 28.10.1904 (wie Anm.6)). Eine eigene Qualität hatte, wie geschildert, der Mayer vom Papst verliehene Gregorius-Orden.

109 Heuss (wie Anm. 2) S. 160.

110 Ogger (wie Anm. 2) S. 46.

111 Heuss (wie Anm. 2) S. 155.

112 Vgl. dazu Rudzinsky (wie Anm.2) S. 83. 